HIP-2011-09/TH

BI-TP 2011/11

\title{
Frequency and wave number dependence of the shear correlator in strongly coupled hot Yang-Mills theory
}

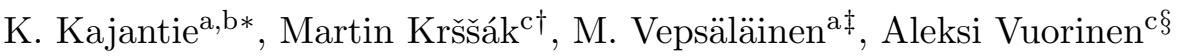 \\ ${ }^{a}$ Department of Physics, P.O.Box 64, FI-00014 University of Helsinki, Finland \\ ${ }^{\mathrm{b}}$ Helsinki Institute of Physics, P.O.Box 64, FI-00014 University of Helsinki, Finland \\ ${ }^{\mathrm{c}}$ Faculty of Physics, University of Bielefeld, D-33501 Bielefeld, Germany
}

\begin{abstract}
We use AdS/QCD duality to compute the finite temperature Green's function $G(\omega, k ; T)$ of the shear operator $T_{12}$ for all $\omega, k$ in hot Yang-Mills theory. The goal is to assess how the existence of scales like the transition temperature and glueball masses affects the correlator computed in the scalefree conformal $\mathcal{N}=4$ supersymmetric Yang-Mills theory. We observe sizeable effects for $T$ close to $T_{c}$ which rapidly disappear with increasing $T$. Quantitative agreement of these predictions with future lattice Monte Carlo data would suggest that QCD matter in this temperature range is strongly interacting.
\end{abstract}

*keijo.kajantie@helsinki.fi

${ }^{\dagger}$ krssak@physik.uni-bielefeld.de

${ }^{\ddagger}$ mikko.vepsalainen@helsinki.fi

$\S$ vuorinen@physik.uni-bielefeld.de 


\section{Introduction}

There is a standard framework for computing Green's functions in supersymmetric $\mathcal{N}=4$ Yang-Mills theory using AdS/CFT duality [1, 2, 3, 4, 5, 6, 7]. In [8], this was carried out explicitly over the entire $\omega, k$ plane for an operator coupling to a scalar field. The specific goal was to compute the real static $(\omega=0)$ Green's function both directly and by integrating over $\omega$. The result was then Fourier transformed to a spatial Green's function and compared with a computation in next-to-leading order QCD perturbation theory [9, 10, see also [11, 12.

The previous calculation was carried out in a scalefree theory. The purpose of this article is to see what effects arise from $\Lambda_{\mathrm{QCD}}$, i.e., scale invariance breaking in $\mathrm{SU}\left(N_{c}\right)$ Yang-Mills theory. Physically this manifests itself in the existence of glueballs and a first order phase transition ${ }^{1}$. The predictions are quantitative and it is suggested that their comparison with lattice Monte Carlo data may give a better handle on verifying the strongly interacting nature of QCD matter in the temperature range $T_{c} \ldots 10 T_{c}$. There is definite phenomenological evidence for this (magnitude of pressure, small shear viscosity, jet quenching), but no definite nonperturbative test [14]. In fact, how can the system be strongly interacting while so many lattice results [15] agree with ideal gas? One concrete test [5, 16] would be to verify if the shear spectral function has a transport peak at $\omega=0$ or not; our calculation addresses this question.

There are many candidate gravity duals of $\mathrm{SU}\left(N_{c}\right)$ Yang-Mills theory. The dual we want to apply is improved holographic QCD (IHQCD) as developed in [17, 18, 19, 20]. This is a bottom-up Anti de Sitter (AdS) gravity+dilaton model which combines elegantly asymptotic freedom in the ultraviolet (UV) with a given beta function and confinement in the infrared (IR). IHQCD has to be solved numerically and we can so far only compute the imaginary part of the Green's function the case $k=0$ as a function of $\omega$. Since we want to treat the entire $\omega, k$ plane, and for increased transparency, we shall in this note also use a simplified version of IHQCD for which the gravity background is analytically known [18. It contains qualitatively the relevant features of IHQCD and computations in the overlapping region confirm that it gives good idea of what one can expect of scale invariance breaking effects on Green's functions in $\mathrm{SU}\left(N_{c}\right)$ Yang-Mills theory.

IHQCD contains the background metric and the scalar dynamically coupled, and fluctuation analysis should be carried out taking this into account [21, 22, 23]. The transverse tensor mode $\left(T_{12}\right.$ with $\mathbf{k}=(0,0, k)$; also called shear mode or scalar mode) does not couple to other ones and we thus consider only this case and study the retarded Green's function

$$
G(\omega, k ; T)=\int_{-\infty}^{\infty} d t d^{3} x e^{i \omega t-i k x^{3}}\left\langle i \theta(t)\left[T_{12}(t, \mathbf{x}), T_{12}(0, \mathbf{0})\right]\right\rangle_{T}=\int_{-\infty}^{\infty} \frac{d \omega^{\prime}}{\pi} \frac{\rho\left(\omega^{\prime}, k\right)}{\omega^{\prime}-\omega-i \epsilon}
$$

On scales and notation: in IHQCD, the QCD-like scale $\Lambda$ arises as an integration constant of the dynamical dilaton field equation, giving the $z$ dependence of the dilaton field, $z$ being the fifth dimension. In the model studied here, it is introduced as a constant of dimension 1 by the $z$ dependence of the ansatz (15). The spin 2 glueball spectrum (squared) then will be $4 \Lambda^{2}(2+n), n=0,1,2, .$. (analytical), and the transition temperature $T_{c}=0.400 \Lambda$ (numerical

\footnotetext{
${ }^{1} \mathrm{~A}$ computation in a non-conformal theory but without a phase transition has been carried out in 13 .
} 
result). Energy (and wave number) can be made dimensionless by scaling with either $T$ or $\Lambda$ and we shall define

$$
\omega=\pi T \tilde{\omega}=2 \Lambda \hat{\omega} \approx 5.00 T_{c} \hat{\omega},
$$

similarly for $k$. As the dimensionless fifth coordinate we shall use

$$
y=\Lambda^{2} z^{2} .
$$

Unless specifically noted, results for the retarded Green's function $G$ are given without writing explicitly the dimensionless factor $\mathcal{L}^{3} /\left(4 \pi G_{5}\right)$, where $\mathcal{L}$ is the AdS radius and $G_{5}$ the $5 \mathrm{dim}$ gravitational constant.

\section{Gravity dual}

\subsection{IHQCD}

IHQCD is based on the gravity + scalar action, in standard notation,

$$
S=\frac{1}{16 \pi G_{5}}\left\{\int d^{5} x \sqrt{-g}\left[R-\frac{4}{3}\left(\partial_{\mu} \phi\right)^{2}+V(\phi)\right]-\int d^{4} x \sqrt{-\gamma}\left[2 K+\frac{6}{\mathcal{L}}+\frac{\mathcal{L}}{2} R(\gamma)\right]\right\} .
$$

The background, with boundary at $z=0$, is chosen to be

$$
d s^{2}=b^{2}(z)\left[-f(z) d t^{2}+d \mathbf{x}^{2}+\frac{d z^{2}}{f(z)}\right]
$$

where $b(z), f(z), \phi(z)$ are solutions of the first order system

$$
\begin{aligned}
\dot{W} & =4 b W^{2}-\frac{1}{f}\left(W \dot{f}+\frac{1}{3} b V\right), \quad W=-\dot{b} / b^{2}, \\
\dot{b} & =-b^{2} W, \\
\dot{\lambda} & =\frac{3}{2} \lambda \sqrt{b \dot{W}}, \\
\ddot{f} & =3 \dot{f} b W,
\end{aligned}
$$

(see Appendix A of [19]). The coupling of the boundary theory is associated with $\lambda(z)=e^{\phi(z)}$, and a specific combination of the fields, arising in connection of solving Einstein's equations $(\dot{b}=d b / d z)$,

$$
\beta(\lambda)=\frac{\dot{\lambda}}{\dot{b} / b},
$$

is associated with the beta function of the boundary theory. An important general property of the solutions is that $b$ and $f$ are monotonically decreasing and $\lambda$ is monotonically increasing as functions of $z$.

The solutions have to satisfy the following properties. In the UV, $z \rightarrow 0$,

$$
b(z) \rightarrow \frac{\mathcal{L}}{z} .
$$


Imposing asymptotic freedom to leading order, one then has,

$$
\beta(\lambda)=\frac{\dot{\lambda}}{\dot{b} / b}=-b_{0} \lambda^{2}=-z \frac{d \lambda}{d z},
$$

implying that the leading term of $\lambda(z)$ near the boundary is

$$
b_{0} \lambda(z)=\frac{1}{L}, \quad L \equiv \log \frac{1}{\Lambda z},
$$

$\Lambda=$ constant of integration. This is how the dimensional transmutation of $\mathrm{SU}\left(N_{c}\right) \mathrm{YM}$ theory appears in the gravity dual. Note that on the boundary $\lambda \rightarrow 0$ but $\phi \rightarrow-\infty$.

Including more powers of $\lambda$ in the beta function, one can derive expansions in $\lambda$ for all the fields. For the record, we have collected some of them in Appendix A.

In the IR, for $z \rightarrow \infty$, the crucial confinement criterion is that out of the many different solutions of Einstein's equations (8) one should choose the one satisfying

$$
\beta(\lambda)=-\frac{3}{2} \lambda\left[1+\frac{3(\alpha-1)}{4 \alpha} \frac{1}{\log \lambda}+\mathcal{O}\left(\frac{1}{\log ^{2} \lambda}\right)\right] .
$$

The parameter $\alpha>1$ here is connected with the IR behavior of all the other background functions so that, for example,

$$
b(z) \rightarrow e^{-(\Xi z)^{\alpha}}(\Xi z)^{p}, \quad \Xi=\text { number } \times \Lambda, \quad p=\text { number. }
$$

The appropriate IR relations are summarised in Appendix B. We shall next choose $p=-1$ to extend the form to all $z$ and $\alpha=2$ to have a glueball spectrum with $m^{2} \sim$ integer [25].

\subsection{Model: approximate version of IHQCD}

Our model for simulating the essential properties of IHQCD is defined by a simple ansatz [18] for $b(z)$ :

$$
b(z)=\frac{\mathcal{L}}{z} \exp \left(-\frac{1}{3} \Lambda^{2} z^{2}\right)=\frac{\mathcal{L} \Lambda}{\sqrt{y}} \exp \left(-\frac{1}{3} y\right),
$$

where we often set $\mathcal{L}=1$ and where we use the dimensionless distance variable $y$ in (3).

Starting from (15) one can derive all the other bulk fields. First, integrating (7) leads to

$$
\phi^{\prime}(y)=\frac{\lambda^{\prime}(y)}{\lambda(y)}=\frac{1}{2} \sqrt{1+\frac{9}{2 y}},
$$

which integrates to

$$
\lambda(y) / \lambda_{0}=\exp \left(\frac{1}{2} \sqrt{y\left(y+\frac{9}{2}\right.}\right)\left(\sqrt{y}+\sqrt{y+\frac{9}{2}}\right)^{9 / 4} .
$$

Now that $\lambda(y)$ is known, the $\beta$ function as specified by IHQCD is obtained:

$$
\beta(\lambda)=\frac{\lambda \phi^{\prime}(y)}{b^{\prime}(y) / b(y)}=-\frac{3}{2} \lambda \frac{\sqrt{1+9 /(2 y)}}{1+3 /(2 y)} .
$$


In the IR this goes like

$$
\beta(\lambda)=-\frac{3}{2} \lambda\left(1+\frac{3}{4 y}+\ldots\right)=-\frac{3}{2} \lambda\left(1+\frac{3}{8 \log \lambda}+\ldots\right)
$$

and thus satisfies explicitly the IHQCD confinement criterion. In the UV, $\lambda(y)$ goes to $\lambda(0)(1+$ $3 \sqrt{y / 2}+9 y / 4+\ldots)$, i.e., this ansatz does not produce a $\lambda(y)$ vanishing logarithmically at the boundary. The far UV region is inessential for the treatment of thermodynamics in IHQCD 24] and the absence of the "spurious logarithms" [26] simplifies the AdS analysis significantly.

To get $f(z)$, we note that (8) integrates to $(C$ is a constant)

$$
\dot{f}(z)=C / b^{3}(z) .
$$

The greatest utility of the ansatz (15) lies in that this is integrable in closed form. Using $f(0)=1, f\left(y_{h}\right)=0$, one has

$$
\begin{aligned}
f(y) & =1-\frac{(y-1) e^{y}+1}{\left(y_{h}-1\right) e^{y_{h}}+1}=\frac{y_{h}-1-(y-1) e^{y-y_{h}}}{y_{h}-1+e^{-y_{h}}} \\
& =\frac{1}{y_{h}-1+e^{-y_{h}}} \sum_{n=1}^{\infty} \frac{y_{h}+n-1}{n !}(-1)^{n+1}\left(y_{h}-y\right)^{n} .
\end{aligned}
$$

Finally, the scalar potential $V(\lambda)$ can be solved from (6) with the answer (keeping $\mathcal{L}$ here to show where the dimensions of $V$ come from)

$$
V(\lambda(y))=\frac{12}{\mathcal{L}^{2}} \cdot e^{\frac{2}{3}} y\left[\left(\frac{1}{3} y^{2}+\frac{5}{6} y+1\right) f(y)-\left(\frac{1}{2}+\frac{1}{3} y\right) y f^{\prime}(y)\right] .
$$

In the vacuum $f=1, f^{\prime}=0$ and, using (17), the potential at large $\lambda$ is seen to behave as

$$
V(\lambda(y))=\frac{12}{\mathcal{L}^{2}} \lambda^{4 / 3} \sqrt{\log \lambda}(1+\mathcal{O}(1 / \log \lambda)),
$$

again in agreement with the confining potential criteria in IHQCD. A conceptual problem with $V(\lambda)$ in $(22)$ is its dependence on $y_{h}$. However, what matters for solving the background is the range $0<y<y_{h}$, and in this range the potentials computed from (22) for $f=1$ and $f=f(y)$ are very close to each other. They differ maximally at $y=y_{h}$ and this difference is maximally $4.7 \%$ if $y_{h}=0.79$.

\subsubsection{Thermodynamics}

To assess the relevance of the model $b(z)$ in (15), we first work out its thermodynamics. From $4 \pi T=-\dot{f}\left(z_{h}\right)$ and 21 , one obtains the entire thermodynamics parametrised by $y_{h}$ (including now the factor $\mathcal{L}$ in $b(z)$ ):

$$
\begin{aligned}
T\left(y_{h}\right) & =\frac{\Lambda}{2 \pi} \frac{y_{h}^{3 / 2}}{y_{h}-1+e^{-y_{h}}} \equiv 2 \Lambda \hat{T}, \\
s\left(y_{h}\right) & =\frac{\mathcal{L}^{3}}{4 G_{5}} \Lambda^{3} y_{h}^{-3 / 2} e^{-y_{h}}, \\
p\left(y_{h}\right) & =\int_{y_{h}}^{\infty} d x\left[-T^{\prime}(x)\right] s(x) .
\end{aligned}
$$


Using (17), we may replace $y_{h}$ by $\lambda_{h}$, which is used in [18]. The overall structure of $T$ is such that it diverges both for small $y_{h}\left(\pi T \rightarrow \Lambda / \sqrt{y_{h}}\right.$, the big black hole limit $)$ and for large $y_{h}$ $\left(\pi T \rightarrow \frac{1}{2} \Lambda \sqrt{y_{h}}\right.$, the small BH limit). Thus, $T\left(y_{h}\right)$ has in between a minimum which lies at

$$
y_{h}=2.149, \quad T_{\min }=0.3962 \Lambda .
$$

For $T>T_{\min }$ the system is in a deconfined plasma phase with pressure $\sim N_{c}^{2}$, but actually in a part of this range, $T_{c}>T>T_{\min }$, the plasma phase is metastable. The stable phase for $0<T<T_{c}$ is a thermal glueball gas phase with pressure $\sim N_{c}^{0}$, i.e., in this approach $p=0$. The transition temperature $T_{c}$ is where the pressure vanishes, which happens at

$$
y_{h}=y_{c}=1.6863, \quad T_{c}=0.4000 \Lambda=1.0096 T_{\min } .
$$

In the UV, where $y_{h} \rightarrow 0$ and $\pi T \rightarrow \Lambda / \sqrt{y_{h}}$,

$$
\frac{p}{T^{4}} \rightarrow \frac{\mathcal{L}^{3}}{4 G_{5}} \frac{\pi^{3}}{4}
$$

independent of the IR scale $\Lambda$. This can be used to fix the dimensionless combination $\mathcal{L}^{3} /\left(4 G_{5}\right)$, which then becomes $\sim N_{c}^{2}$. For example, in the present AdS/QCD context one may match at some large $T \gg T_{c}$ to the pressure of weakly interacting gluon gas, $p / T^{4}=\pi^{2} N_{c}^{2} / 45$. Weakly interacting $\mathcal{N}=4$ supersymmetric YM matter has $p / T^{4}=\pi^{2} N_{c}^{2} / 6$ while the strongly interacting one has $p / T^{4}=\pi^{2} N_{c}^{2} / 8$ with

$$
\frac{\mathcal{L}^{3}}{4 \pi G_{5}}=\frac{N_{c}^{2}}{2 \pi^{2}}
$$

The resulting thermodynamics is plotted in Fig. 1. The interaction measure is broader than that of pure YM at large $N_{c}$ and the latent heat smaller. The latent heat is related to the large $T$ asymptotics by

$$
L=\frac{\epsilon_{c}}{T_{c}^{4}}=\frac{s_{c}}{T_{c}^{3}}=\left.\frac{32}{3} \frac{\left(y_{c}-1+e^{-y_{c}}\right)^{3}}{y_{c}^{6}} e^{-y_{c}} \frac{\epsilon}{T^{4}}\right|_{T \rightarrow \infty} .
$$

The smallness of $L$ is due to the fact that the $y_{c}$-dependent term at $y_{c}=1.6863$ is already considerably reduced from its maximum value $1 / 8$. There is no parameter with which one could tune $L$ to a larger value.

Finally, the sound velocity is given by

$$
c_{s}^{2}=\frac{1}{3+2 y_{h}} \frac{3-y_{h}-\left(3+2 y_{h}\right) e^{-y_{h}}}{y_{h}-1+e^{-y_{h}}}=\frac{1}{3}-\frac{1}{(3 \pi \hat{T})^{2}}+. .
$$

At $T_{c} c_{s}^{2}=0.024$. Note that the Chamblin-Reall background used in [13] has a constant sound velocity and does not have a phase transition, since for it always $\epsilon=$ constant $\times p$. 

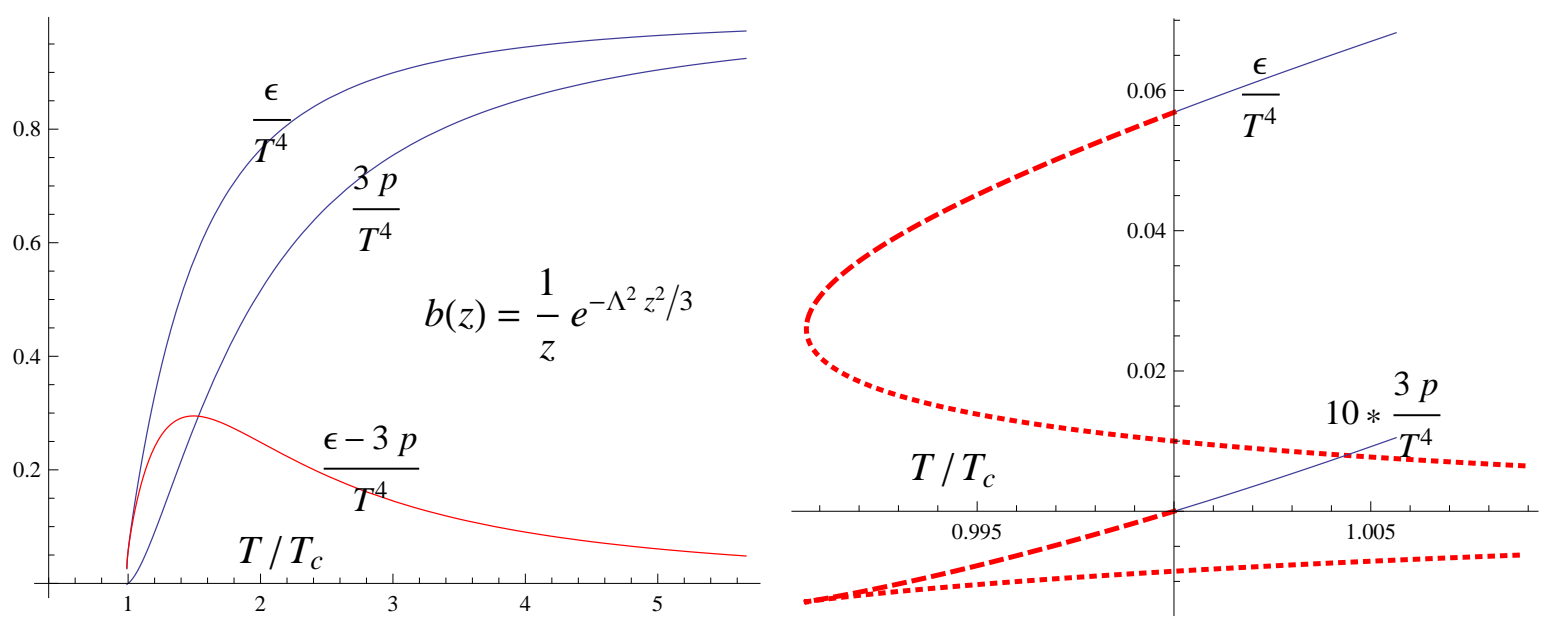

Figure 1: Thermodynamics from the model $b(z)$. The curves are normalised to their $T \rightarrow \infty$ asymptotic values, which are $\sim N_{c}^{2}$. At $T_{c}$ there is a weak first order transition with $\epsilon_{c} / T_{c}^{4}=0.06$. The right panel shows the region around $T_{c}$ with the supercooled (dashed) and unstable (dotted) phases. The curve for pressure is multiplied by 10 for clarity.

\subsubsection{Glueball masses}

Glueball masses are obtained as poles of Green's function and we shall later see how this goes through. Effectively, this leads to a Schrödinger-type equation,

$$
-\psi^{\prime \prime}(z)+V_{\mathrm{Schr}}(z) \psi(z)=m^{2} \psi(z),
$$

where, for the background (5) and for scalar glueballs,

$$
V_{\mathrm{Schr}}=\frac{3}{2}\left(\frac{\ddot{b}}{b}+\frac{\dot{b}^{2}}{2 b^{2}}\right)+\frac{\ddot{X}}{X}+3 \frac{\dot{b}}{b} \frac{\dot{X}}{X}, \quad X \equiv \frac{\beta(\lambda)}{3 \lambda} ;
$$

for tensor glueballs the $X$-terms are missing. With the above explicit expressions it is trivial to evaluate the potentials. Scaling out $\Lambda$ by writing $V / \Lambda^{2} \rightarrow V, \Lambda z \rightarrow z$, the tensor potential is

$$
V_{t}(z)=\frac{15}{4 z^{2}}+2+z^{2}
$$

and the scalar one

$$
V_{s}(z)=V_{t}(z)+\frac{1}{\left(3+2 z^{2}\right)^{2}\left(9+2 z^{2}\right)^{2}}\left(-\frac{2187}{z^{2}}-5346-1224 z^{2}+216 z^{4}+48 z^{6}\right) .
$$

The tensor equation is analytically solvable [25] and leads to the mass spectrum

$$
m_{T}^{2} / \Lambda^{2}=4(n+2), \quad n=0,1,2, \ldots
$$


Solving numerically the Schrödinger equation, one finds the scalar glueball mass spectrum

$$
m_{S}^{2} / \Lambda^{2}=6.27,10.27,14.25, \ldots
$$

The lowest tensor and scalar states are in the ratio $m_{T 1} / m_{S 1}=1.13$ (in the full IHQCD this ratio is 1.36 [20]), but the $m^{2}$ spacing is about $4 \Lambda^{2}$ also for scalars. In the full IHQCD the ratio $m_{S 2} / m_{S 1}=1.61$ while here it is 1.28 .

\section{Fluctuation equation}

To calculate correlators [1, 2, 3, 4] of the operator $T_{12}$ in the background (5) one has to solve the fluctuation equation

$$
\ddot{h}+\frac{d}{d z} \log \left(b^{3} f\right) \dot{h}+\left(\frac{\omega^{2}}{f^{2}}-\frac{\mathbf{k}^{2}}{f}\right) h \equiv \ddot{h}+P \dot{h}+Q h=0 .
$$

Here $h \equiv h_{K}(z), K=(\omega, \mathbf{k})$, and

$$
h(x, z)=\int \frac{d^{4} K}{(2 \pi)^{4}} e^{-i \omega t+i \mathbf{k} \cdot \mathbf{x}} h_{0}(K) h_{K}(z), \quad h_{K}(0)=1,
$$

is the fluctuation of the 12 component of the background metric: $g_{12}=b^{2}(z)\left(1+h\left(t, x^{3}, z\right)\right)$. This tensor fluctuation does not mix with other ones. In IHQCD, the scalar one does and the fluctuation equation permitting computations of the Green's function of the scalar operator $F_{\mu \nu}^{2}$ will contain more terms [21, 22, 23].

The characteristic exponents of 39 at $z=z_{h}$ are $\pm i \omega / \dot{f}_{h}$. Eq. (39) should be solved so that at the horizon (infalling boundary condition, $d_{i}$ are calculable coefficients)

$$
h_{K}\left(z \rightarrow z_{h}\right)=\left(z-z_{h}\right)^{i \omega / \dot{f}_{h}}\left[1+d_{1}\left(z-z_{h}\right)+d_{2}\left(z-z_{h}\right)^{2}+\ldots\right]
$$

and that at the boundary, $h_{K}(0)=1$ (correct normalisation). These solutions will satisfy

$$
h_{-K}(z)=h_{K}^{*}(z) .
$$

Given a solution, a computation along the lines of [27] shows that the retarded Green's function is obtained from

$$
G(K)=\frac{1}{16 \pi G_{5}}\left[f b^{3} \dot{h}_{K} h_{-K}+(\text { counter terms }) \times h_{K} h_{-K}\right],
$$

where the $h_{K} h_{-K}=h_{K} h_{K}^{*}$ terms with unspecified coefficient are real.

For the imaginary part of $G$, the spectral function, the situation is particularly simple:

$$
\operatorname{Im} G(k) \equiv \rho(\omega, k)=\frac{1}{16 \pi G_{5}} f b^{3} \operatorname{Im} \dot{h}_{K} h_{-K}=\frac{1}{16 \pi G_{5}} f b^{3} W\left(h_{K}, h_{K}^{*}\right) /(2 i),
$$


where one has noted that $h_{K}$ and $h_{K}^{*}$ are two independent solutions of (39) and that their Wronskian arises when $\operatorname{Im} G$ is evaluated. However, the Wronskian of any two linearly independent solutions of 39 is, integrating $\dot{W} / W=-P$,

$$
W\left(\phi_{1}, \phi_{2}\right) \equiv \phi_{1} \dot{\phi}_{2}-\phi_{2} \dot{\phi}_{1}=\frac{W_{0}(K)}{b^{3} f},
$$

where $W_{0}$ is a $z$ independent constant (but will depend on $K=\omega, k$ ). Thus the result in (44) is a ratio of two Wronskians and independent of $z$.

To find the constant $W_{0}$ for the Wronskian $W\left(h_{K}, h_{K}^{*}\right)$ we simply evaluate it in the limit $z \rightarrow z_{h}$ using the expansion (41) and find

$$
W\left(h_{K}, h_{K}^{*}\right)=\frac{2 i \omega}{f b^{3}} b^{3}\left(z_{h}\right) .
$$

The result thus automatically contains the entropy density, which in IHQCD is

$$
s(T)=\frac{A}{4 G_{5}}=\frac{1}{4 G_{5}} b^{3}\left(z_{h}\right) .
$$

However, so far this is for a solution normalised as in (41), which leads to some value $h_{K}(0)$ at the boundary. Correcting for the normalisation, the result is

$$
\rho(\omega, k)=\frac{1}{4 \pi} s(T) \frac{\omega}{\left|h_{K}(0)\right|^{2}} .
$$

A practical note on the application of this formula: in it $h_{K}(z)$ arises from numerical integration which cannot be extended to $z=0$ since this is a singular point of the equation. However, in $(48) h_{K}(z)$ has to be evaluated precisely at $z=0$ and this value has to be found by extrapolation. Numerical effect is significant at large $\omega$, where $\left|h_{K}(0)\right| \sim 1 / \omega^{3 / 2}$.

We shall also need the real part, for example, the static Green's function $G(0, k)$ is purely real. For the real part one has to make further assumptions, corresponding to subtractions in the dispersion relation (1). The standard procedure here [3] is to expand the solution $h_{K}(z)$ computed with the boundary condition (41) in terms of the two independent solutions at $z=0$ :

$$
h_{K}(z)=A(K) \phi_{u}(z, K)+B(K) \phi_{n}(z, K)
$$

where

$$
\begin{aligned}
\phi_{n} & =z^{4}\left(1+c_{1} z^{2}+c_{4} z^{4}+\ldots\right), \\
\phi_{u} & =1+f_{1} z^{2}+f_{3} z^{6}+\ldots+c \log z \phi_{n}(z, K) .
\end{aligned}
$$

In IHQCD the expansion coefficients will contain logarithmic UV coefficients of the type in Appendix A [17]. Noting that the normalisation factor $h_{K}(0)$ in $(48)$ is $A(K)$ the result (43) can be evaluated using

$$
\begin{aligned}
\frac{h_{K}^{*} \dot{h}_{K}}{z^{3}\left|h_{K}(0)\right|^{2}} & =\frac{1}{z^{3}|A|^{2}}\left(A^{*} \phi_{u}+B^{*} \phi_{n}\right)\left(A \dot{\phi}_{u}+B \dot{\phi}_{n}\right) \\
& =\frac{2 f_{1}}{z^{2}}+4 c \log z+c+2 f_{1}^{2}+4 \frac{B(K)}{A(K)}+\mathcal{O}\left(z^{2}\right) \\
& =\frac{\omega^{2}-k^{2}}{2 z^{2}}-\frac{\left(\omega^{2}-k^{2}\right)^{2}}{16}(4 \log z-1)+4 \frac{B(K)}{A(K)}+\mathcal{O}\left(z^{2}\right),
\end{aligned}
$$


where the last form applies for conformal expansions (50). The scheme is to neglect the real divergent terms + constant in (51) and to evaluate the entire Green's function from

$$
G(K)=\frac{\mathcal{L}^{3}}{4 \pi G_{5}} \frac{B(K)}{A(K)}, \quad \frac{B(K)}{A(K)}=-\frac{W\left(h_{K}, \phi_{u}\right)}{W\left(h_{K}, \phi_{n}\right)}=-\frac{h_{K} \dot{\phi}_{u}-\phi_{u} \dot{h}_{K}}{h_{K} \dot{\phi}_{n}-\phi_{n} \dot{h}_{K}} .
$$

Equivalently, one may say that this is a way to derive the counter terms in (43] [28]. In [8] this scheme was tested by evaluating the purely real static Green's function $G(0, k)$ also by an $\omega$-integral over the complex $G(\omega, k)$. It also gave correctly the real and imaginary parts on the light cone, for $G(k, k)$.

Since $G(K)$ in $(52)$ is given as a ratio of two Wronskians, it is independent at what $z$ it is evaluated, to the extent $h_{K}, \phi_{u}, \phi_{n}$ are correct solutions. Since $h_{K}$ is integrated numerically, it certainly is a solution. In practice the accuracy and constancy then depends on how many terms are included in small- $z$ expansions of $\phi_{u}, \phi_{n}$. If the numerical integration of $h_{K}$ from (39) is extended down to some small $\epsilon$, the ratio of Wronskians can be evaluated at this $\epsilon$. This is to be contrasted with (48) in which $h_{K}(0)$ has to be extrapolated to $z=0$ for accuracy. Of course, (48) only gives the imaginary part while both the real and imaginary parts are obtained from (52).

\subsection{Equations for the model}

Next, we specialize to the model defined in subsection 2.2. In terms of $y=\Lambda^{2} z^{2}$ the fluctuation equation 39 becomes

$$
\phi_{K}^{\prime \prime}(y)+P(y) \phi_{K}^{\prime}(y)+Q(y) \phi_{K}(y)=0 .
$$

where

$$
\begin{aligned}
P(y)=\frac{d}{d y} \log \left(b^{3} f \sqrt{y}\right) & =-\frac{1}{y}-1+\frac{y}{y-1+\left(1-y_{h}\right) e^{y_{h}-y}}, \\
Q(y) & =\frac{\hat{\omega}^{2}}{y f^{2}(y)}-\frac{\hat{k}^{2}}{y f(y)},
\end{aligned}
$$

where the dimensionless $\hat{\omega}, \hat{k}$ were defined in $(2)$.

The equation (53) is to be solved so that at the horizon (the $y-y_{h}$ term is for $k=0$ )

$$
\phi_{K}(y)=\left(y-y_{h}\right)^{p}\left[1+\frac{p\left(1+y_{h}\right)-2 p\left(2+y_{h}\right)}{2 y_{h}(1+2 p)}\left(y-y_{h}\right)+\mathcal{O}\left(\left(y-y_{h}\right)^{2}\right)\right] .
$$

where

$$
p=\frac{-i \omega}{4 \pi T}=i \frac{\hat{\omega}}{\sqrt{y_{h}} f^{\prime}\left(y_{h}\right)}=-i \frac{\hat{\omega}}{y_{h}^{3 / 2}}\left(y_{h}-1+e^{-y_{h}}\right) .
$$

For correct normalisation, the solution is divided by $\phi_{K}(0)$.

The Green's function now is

$$
G(K)=\frac{2 \Lambda}{16 \pi G_{5}}\left[f b^{3} \sqrt{y} \phi_{K}^{\prime} \phi_{-K}+\text { counter terms } \cdot \phi_{K} \phi_{-K}\right] .
$$


For the spectral function one again needs the Wronskian of the two independent solutions at the horizon,

$$
W\left(\phi_{K}, \phi_{K}^{*}\right)=\frac{2 i \hat{\omega}}{f b^{3} \sqrt{y}} b^{3}\left(y_{h}\right),
$$

and the result (48) is unchanged. For the real part and evaluation of the counter terms in (58) the expansions around $y=0$ have to be worked out. One writes

$$
\phi_{K}(y)=A(K) \phi_{u}(y, K)+B(K) \phi_{n}(y, K),
$$

where

$$
\begin{gathered}
\phi_{n}(y, K)=y^{2}\left[1+\frac{1}{3}\left(2+k^{2}-\hat{\omega}^{2}\right) y+\ldots\right] \\
\phi_{u}(y, K)=1+\left(\hat{\omega}^{2}-k^{2}\right) y-\frac{1}{2}\left[\left(\hat{\omega}^{2}-\hat{k}^{2}\right)^{2}-\left(\hat{\omega}^{2}-\hat{k}^{2}\right)\right] \log y \cdot \phi_{n}(y)+\mathcal{O}\left(y^{3}\right)+\ldots
\end{gathered}
$$

(note no $y^{2}$ term; it is only in $\phi_{n}$ ). The real counter terms neglected now are

$$
2 \Lambda^{4}\left\{\frac{\hat{\omega}^{2}-\hat{k}^{2}}{y}-\frac{1}{2}\left[\left(\hat{\omega}^{2}-\hat{k}^{2}\right)^{2}-\left(\hat{\omega}^{2}-\hat{k}^{2}\right)\right](2 \log y+1)\right\}
$$

and, solving $A$ and $B$ with Wronskians,

$$
G(K)=\frac{\mathcal{L}^{3}}{4 \pi G_{5}} \cdot \Lambda^{4} \frac{B(K)}{A(K)}
$$

Now the correct dimension 4 comes from $\Lambda^{4}$. In units of $\pi T$, using (24):

$$
\frac{G(K)}{(\pi T)^{4}}=\frac{\mathcal{L}^{3}}{4 \pi G_{5}} \cdot \frac{16\left(y_{h}-1+e^{-y_{h}}\right)^{4}}{y_{h}^{6}} \cdot \frac{B(K)}{A(K)} .
$$

We are interested in the high $T$ phase and, as was derived earlier, this means $T \geq T_{c}=$ $0.4000 \Lambda$ or $y_{h}<y_{c}=1.6863$.

\subsection{Vacuum spectral function and the limit at large $\omega$ or $k$}

To obtain the vacuum Green's function of the model we put $f=1$ in (53) and obtain the equation

$$
y \phi^{\prime \prime}(y)+(-1-y) \phi^{\prime}(y)+\hat{K}^{2} \phi(y)=0, \quad \hat{K}^{2} \equiv \hat{\omega}^{2}-\hat{k}^{2} .
$$

This also gives the leading terms of the $\omega, k \gg \Lambda, T$ limit of the solutions of (53). Understanding this limit analytically is important for numerical accuracy. This equation is the equation for the confluent hypergeometric function with a solution $\phi_{1}$ growing like a power at large $y$ :

$$
\phi_{1}(y)=y^{2} U\left(2-\hat{K}^{2}, 3, y\right) \rightarrow y^{\hat{K}^{2}}\left[1+\hat{K}^{2}\left(2-\hat{K}^{2}\right) y^{-1}+\mathcal{O}\left(y^{-2}\right)\right] .
$$

Its small $y$ expansion is

$$
\phi_{1}(y)=\frac{1}{\Gamma\left(2-\hat{K}^{2}\right)}\left[1+\hat{K}^{2} y+\frac{1}{2} \hat{K}^{2}\left(1-\hat{K}^{2}\right)\left(\log y+\psi\left(2-\hat{K}^{2}\right)+2 \gamma_{E}-\frac{3}{2}\right) y^{2}+\mathcal{O}\left(y^{3}\right)\right] .
$$


The second solution $\phi_{2}(y)=y^{2} L_{\hat{K}^{2}-2}^{(2)}(y) \rightarrow e^{y} y^{-\hat{K}^{2}+1}+.$. , grows exponentially and is unacceptable. If we now decompose the solution $\phi_{1}$ as in (60), $B$ is immediately read as the coefficient of the $y^{2}$ term and $A=1 / \Gamma\left(2-\hat{K}^{2}\right)$. The vacuum (this is vacuum since no $T$ is involved) Green's function then is

$$
G_{\operatorname{vac}}(K)=\Lambda^{4} \cdot \frac{1}{2} \hat{K}^{2}\left(1-\hat{K}^{2}\right)\left[\psi\left(2-\hat{K}^{2}\right)+2 \gamma_{E}-\frac{3}{2}\right] .
$$

Since

$$
\psi(z)=-\gamma_{E}+\sum_{m=0}^{\infty}\left[\frac{1}{m+1}-\frac{1}{m+z}\right],
$$

the result in $(69)$ has poles at

$$
\omega^{2}-k^{2}=4 \Lambda^{2}(m+2) .
$$

This reproduces the tensor glueball mass spectrum in (37), now derived as poles of Green's function. Taking $\omega \rightarrow \omega+i \epsilon$ and plotting $\operatorname{Im} G_{\text {vac }}$ from (69) for finite but small $\epsilon$ one finds Gaussian peaks at glueball masses. In the limit $\epsilon \rightarrow 0$ [29],

$$
\rho_{\mathrm{vac}} \equiv \operatorname{Im} G_{\mathrm{vac}}=\frac{\pi}{32}\left(\omega^{2}-k^{2}\right)\left(\omega^{2}-k^{2}-4 \Lambda^{2}\right) \sum_{m=0}^{\infty} \delta\left(m+2-\frac{\omega^{2}-k^{2}}{4 \Lambda^{2}}\right) .
$$

We shall later use this as a baseline to assess the magnitude of finite $T$ effects (see Eq. (79)).

The above was for vacuum, $f=1$. The asymptotic large $\omega, k$ limit for $f \neq 1$ can be argued as follows. Taking first $\omega>k>0$, the large $\hat{K}^{2}>0$ limit of $\psi$ in 69 is

$$
\psi\left(2-\hat{K}^{2}\right)=\pi \cot \left(\pi \hat{K}^{2}\right)+\log \hat{K}^{2}-\frac{3}{\hat{2} K^{2}}-\frac{13}{12 \hat{K}^{4}}+\ldots
$$

Note now that $\pi \cot (x+i y) \rightarrow \mp i \pi$ if $y \rightarrow \pm \infty$. If now the analytic continuation is performed so that also $\operatorname{Im} \hat{K}^{2} \rightarrow \infty$, then 69 and 73 give

$$
\frac{\operatorname{Im} G(K)}{\Lambda^{4}}=\frac{\pi}{2}\left[\left(\hat{\omega}^{2}-\hat{k}^{2}\right)^{2}-\left(\hat{\omega}^{2}-\hat{k}^{2}\right)+\mathcal{O}(1)\right] .
$$

Both terms are very clearly seen in numerics, but we have been unable to fit a constant (or possibly a logarithmic) term nor compute it analytically.

In contrast, in the static $\omega=0$ case, $\hat{K}^{2}=-\hat{k}^{2}$, the constant term (and higher corrections $\left.\mathcal{O}\left(1 / k^{2}\right)\right)$ can be evaluated. Now Eq. (69) is purely real. Expanding $\psi\left(2+k^{2}\right) \rightarrow \log k^{2}+$ $3 /\left(2 k^{2}\right)-13 /\left(12 k^{4}\right)+\mathcal{O}\left(1 / k^{6}\right)$ in $(69)$ gives a constant term $-5 / 24$. However, this is not the entire constant term, which is crucial for numerical analysis. A careful analysis (see Appendix C) permits one to compute it with the result (116), which is the same as writing (69) in the form

$$
\frac{B}{A} \rightarrow-\frac{1}{2} \hat{k}^{2}\left(1+\hat{k}^{2}\right)\left[\psi\left(2+\hat{k}^{2}\right)+2 \gamma_{E}-\frac{3}{2}\right]+\frac{e^{-y_{h}}}{20\left(y_{h}-1+e^{-y_{h}}\right)} .
$$

Thus there is an overall constant term $-5 / 24+e^{-y_{h}} /\left(20\left(y_{h}-1+e^{-y_{h}}\right)\right) \approx-5 / 24+T_{c}^{4} /(5 T)^{4}$. This is clearly seen in numerics. 

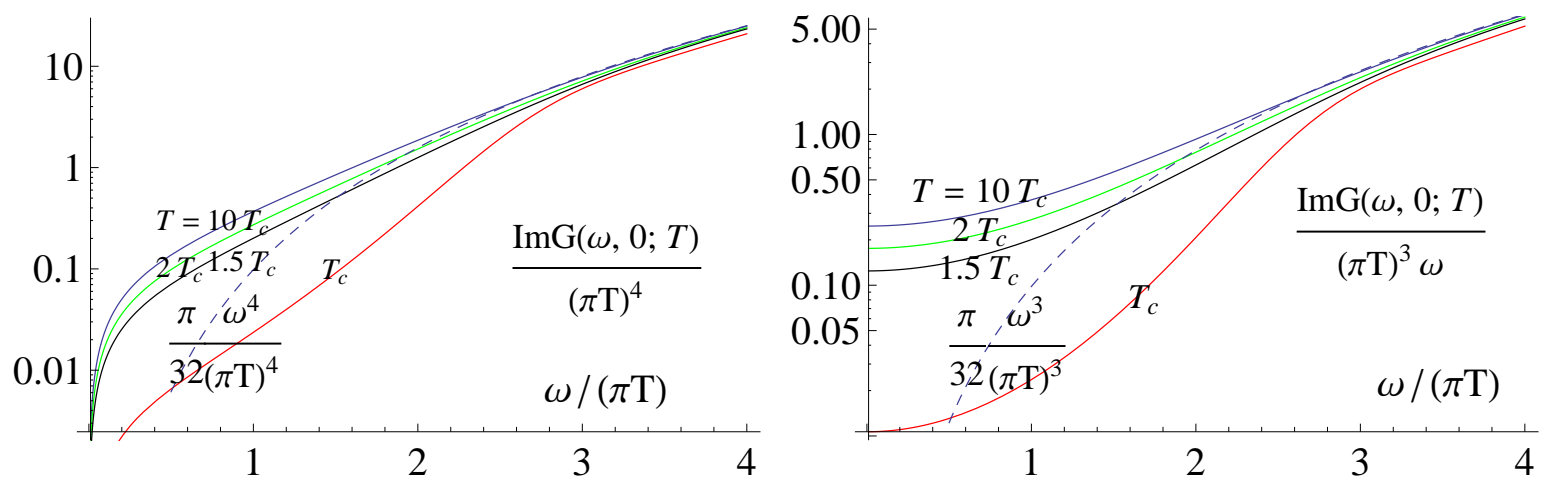

Figure 2: The spectral function $\operatorname{Im} G(\omega, 0 ; T) /(\pi T)^{4}$ in units of $\mathcal{L}^{3} /\left(4 \pi G_{5}\right)$ plotted vs $\omega /(\pi T)$ (left panel) or divided by $\omega /(\pi T)$ (right panel), for the model $(15)$. The dashed line is the leading large- $\omega$ limit.
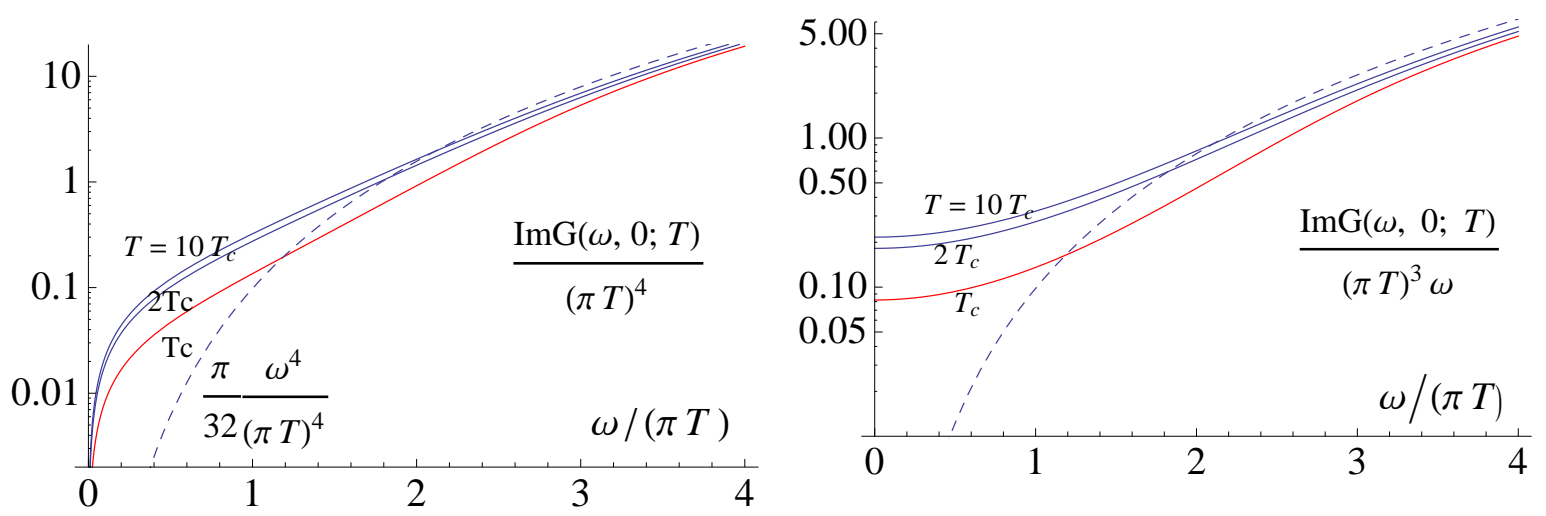

Figure 3: The spectral function $\operatorname{Im} G(\omega, 0 ; T) /(\pi T)^{4}$ in units of $\mathcal{L}^{3} /\left(4 \pi G_{5}\right)$ plotted vs $\omega /(\pi T)$ (left panel) or divided by $\omega /(\pi T)$ (right panel) in exact numerics of IHQCD.

In the thermal theory with $f \neq 1$ the glueballs form a glueball gas with pressure $\mathcal{O}\left(N_{c}^{0}\right)$ and AdS/QCD duality says little about its properties [31, it is the phase at $p=0$ in Fig. 1 . In the high $T$ phase at $T \geq T_{\min }$ the delta function peaks are not seen but still some structure remains (see Figs. 7 and 8 below). The broadening of hadronic states in a thermal ensemble has been studied extensively in connection with quarkonium physics $[32$.

\section{Numerical results}

We are interested in computing the finite temperature retarded Green's function of $T_{12}$ in a QCD-like strongly interacting theory, for various frequences and wave numbers $\mathbf{k}=(0,0, k)$. 

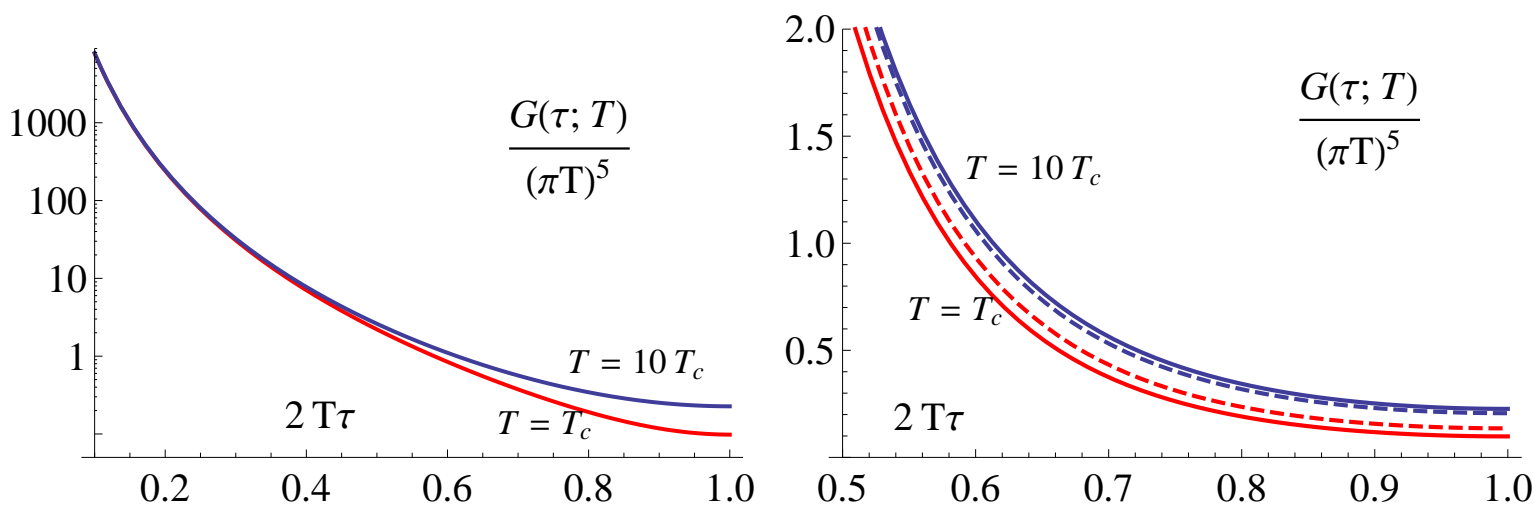

Figure 4: The imaginary time Green's function $G(\tau, k=0)$ computed from 777$)$. The right panel shows the region near $\tau=1 /(2 T)$ on a linear scale, the dashed curves come from full numerical evaluation of IHQCD.

At large $T$, say, $T>10 T_{c}$ one expects the theory to approach a conformal theory with Green's functions explicitly plotted in [4, 5, 8]. In the range $T_{c}$ to $10 T_{c}$ there are important nonconformal effects, reflected in the large value of the interaction measure $(\epsilon-3 p) / T^{4}$. This is the range of $T$ to be studied here.

We remind that the results for $G$ or $\rho=\operatorname{Im} G$ are given without an overall factor of $\mathcal{L}^{3} /\left(4 \pi G_{5}\right)$.

\subsection{Spectral function for $k=0$}

This is clearly a very interesting range due to its relation to viscosity and lattice determinations thereof [33, 34, 35, 36, 37, 39]. Consider first the results for the model (15). As discussed in subsection 3.2 , the large- $\omega$ limit is, measuring both $G$ and $\omega$ in units of $\pi T$,

$$
\frac{\rho_{\mathrm{as}}(\omega, k)}{(\pi T)^{4}}=\frac{\operatorname{Im} G_{\mathrm{as}}(\omega, k ; T)}{(\pi T)^{4}}=\frac{\pi}{32}\left[\left(\tilde{\omega}^{2}-\tilde{k}^{2}\right)^{2}-\frac{25 \cdot 0 T_{c}^{2}}{(\pi T)^{2}}\left(\tilde{\omega}^{2}-\tilde{k}^{2}\right)\right] \theta(\omega-k) .
$$

This is an asymptotic formula and both terms are clearly seen in numerics. However, we do not know the terms of lower order in $\omega^{2}-k^{2}$, but $(76)$ should be applicable only for $\omega^{2}>k^{2}+25.0 T_{c}^{2}$, it is negative at smaller $\omega$.

Results for the model (15) are shown in Fig. 2 together with the leading asymptotic behavior $\pi \omega^{4} / 32$. The spectral function is shown both as such or divided by $\omega$ so that the value at $\omega=0$ is essentially the shear viscosity. One observes a sizeable effect near $T_{c}$ but already at $2 T_{c}$ the curves are very close to the conformal situation, approximated by the $10 T_{c}$ curve. The main $T$-dependent effect is that contained analytically in the asymptotic behavior (76). Even for $T \approx T_{c}$ the nonconformal effects disappear at $\omega \gtrsim 3 T_{c}$; remember that the lightest scalar glueball mass is $\approx 6.2 T_{c}$. 

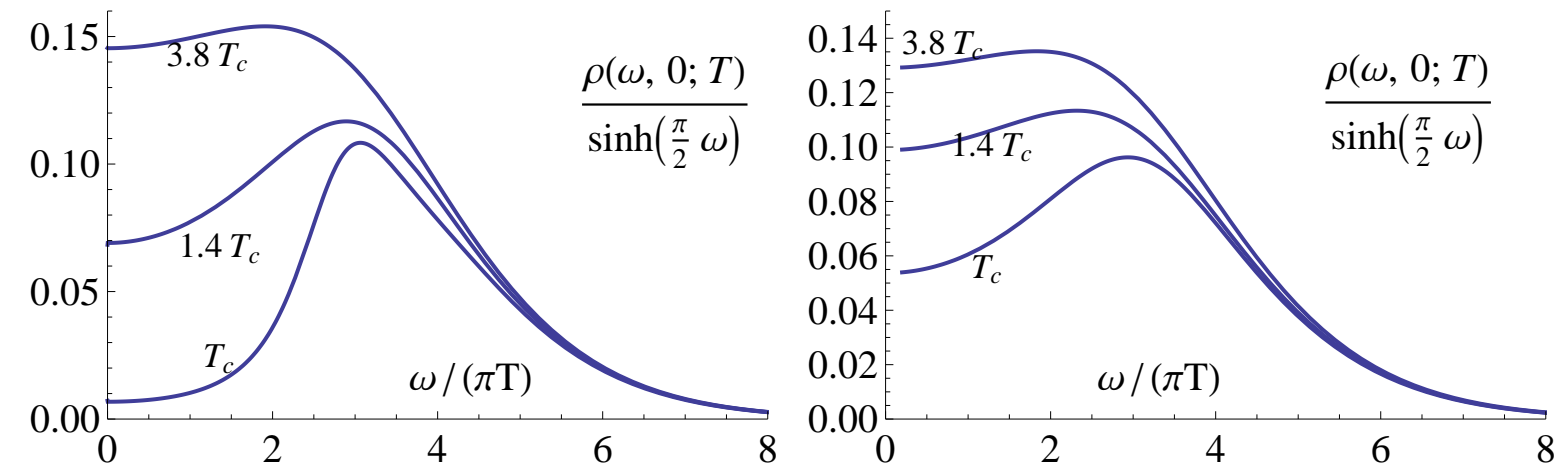

Figure 5: The weighted spectral distribution, integrand of (77), at the middle point $\tau=1 /(2 T)$ for both the model (15) (left) and full numerics of IHQCD (right), at various temperatures.

Consider then the results, shown in Fig. 3, computed from full numeric: ${ }^{2}$ of IHQCD, using the formula (48). This gives only the imaginary part; due to the complicated logarithmic structure of IHQCD (see Appendix A) it will be more complicated to evaluate the Wronskians in (52) and compute the real part also. The overall pattern at $k=0$ is very similar. An interesting detail is that in IHQCD the approach to the asymptotic limit is rather slow, possibly related to the UV logarithms.

The imaginary time $T_{12}$ correlator at $\mathbf{k}=0$ can now be integrated from

$$
G(\tau, 0 ; T)=\int_{0}^{\infty} \frac{d \omega}{\pi} \rho(\omega, 0 ; T) \frac{\cosh \left[(1-2 T \tau) \frac{\pi}{2} \omega\right]}{\sinh \left(\frac{\pi}{2} \omega\right)}
$$

with everything in units of $\pi T$. Note first that in the $\mathcal{N}=4$ conformal case the answer is [8]

$$
G(\tau, 0 ; T)=\frac{3}{4 \pi^{5}}[\zeta(5, T \tau)+\zeta(5,1-T \tau)]+G_{\beta}(T \tau)
$$

where the first terms come from the integration of $\pi \omega^{4} / 32$ and $G_{\beta}$ from $\rho(\omega, 0)-\pi \omega^{4} / 32$. The thermal part $G_{\beta}$ varies very slowly from $G_{\beta}\left(\frac{1}{2}\right)=0.0710$ at the symmetry point to the value 0.13 at $\tau=0$. At $T \tau=\frac{1}{2}$ the vacuum part, i.e., the $\zeta$ function terms, contribute $93 \zeta(5) /\left(2 \pi^{5}\right)=0.1576$ and dominate totally at smaller $\tau$.

The outcome in the nonconformal cases is shown in Fig. 4. The $T=10 T_{c}$ curve is very close to the conformal curve (78). A particularly interesting point is the middle one, $\tau=$ $1 /(2 T)$. Fig. 5 shows the corresponding integrand of $(77)$, in which large $\omega$ values are strongly suppressed, Fig. 6 shows the resulting value of $G(\tau=1 /(2 T))$.

To assess the significance of the result, it is very useful to generate a comparison curve by inserting the vacuum spectral function 72 with delta function peaks at the position of

\footnotetext{
${ }^{2}$ The parameters of the scalar potential were otherwise the same as those in 20] but $V_{0}=1$ instead of $V_{0}=0.04128$; this makes numerics simpler and does not affect the results.
} 


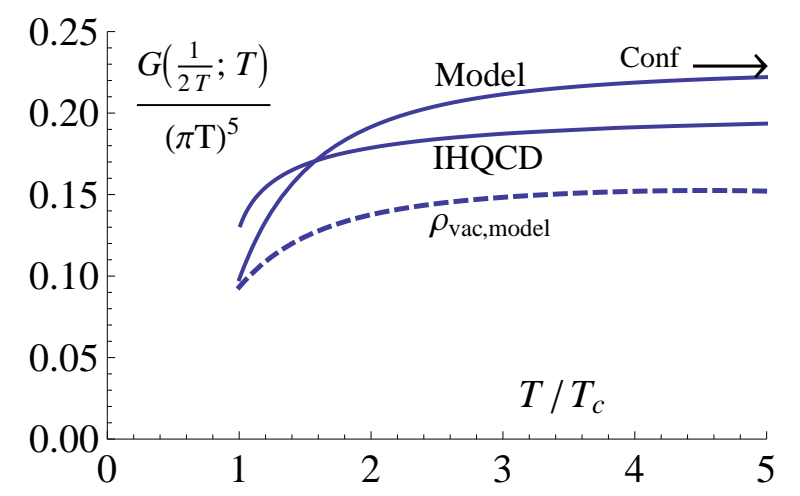

Figure 6: The temperature dependence of $G(1 /(2 T)$ ) for both the model 15 and full numerics of IHQCD. The arrow shows the value for the conformal $\mathcal{N}=4$ theory [8] The dashed curve shows the plot of $(79)$, generated from the $T$ independent vacuum spectral function $(72)$ via the spectral representation (77).

glueball masses to the representation 77$)$ at $\tau=1 /(2 T)$. The answer is $\left(\Lambda=2.5 T_{c}\right)$

$$
\frac{G_{\mathrm{vac}}(\tau=1 /(2 T))}{(\pi T)^{5}}=\frac{1}{2}\left(\frac{2.5 T_{c}}{\pi T}\right)^{5} \sum_{m=0}^{\infty} \frac{\sqrt{m+2}(m+1)}{\sinh \left(2.5 \sqrt{m+2} T_{c} / T\right)} .
$$

The resulting curve is also plotted in Fig. 6, the limit at large $T$ is $93 \zeta(5) /\left(2 \pi^{5}\right)$, the same as for the vacuum curve in 78 . One sees that the imaginary time correlator generated by the vacuum spectral function behaves similarly to the genuine finite $T$ correlator, finite $T$ just enhances the magnitude. The vacuum correlator has nothing like a transport peak at $\omega=0$, it is identically zero up to the lowest glueball mass at $2 \sqrt{2} \Lambda$. Still the outcomes are very similar.

We do not attempt a detailed comparison with lattice Monte Carlo data. However, the results are in qualitative agreement with the plots in [35. An agreement confirmed by careful analysis would support the present picture of strongly interacting QCD matter, but Fig. 6 illustrates the difficulty of making definite conclusions.

Since one knows that the viscosity prediction holds independent of $T$ it is useful to present the data so that this is explicitly seen. Data for the dimensionless quantity

$$
\frac{4 \pi}{s(T)} \frac{\rho(\omega, 0 ; T)}{\omega}
$$

computed for the model (15) in the region of small $\omega$, are presented in Fig. 7, left panel. The normalisation is such that the standard viscosity prediction $\eta / s=1 /(4 \pi)$ corresponds to the value 1 at $\omega=0$. The right panel of Fig. 7 shows the spectral function with the asymptotic behavior $(76)$ subtracted. The subtraction is reliable only for $\omega \gtrsim 5.0 T_{c} /(\pi T)$.

Finally, Fig. 8 shows the subtracted Green's function plotted in non-thermal units, vs. $\omega /(2 \Lambda)$, computed for the model $(15)$. This is what remains of the infinite set of delta function 


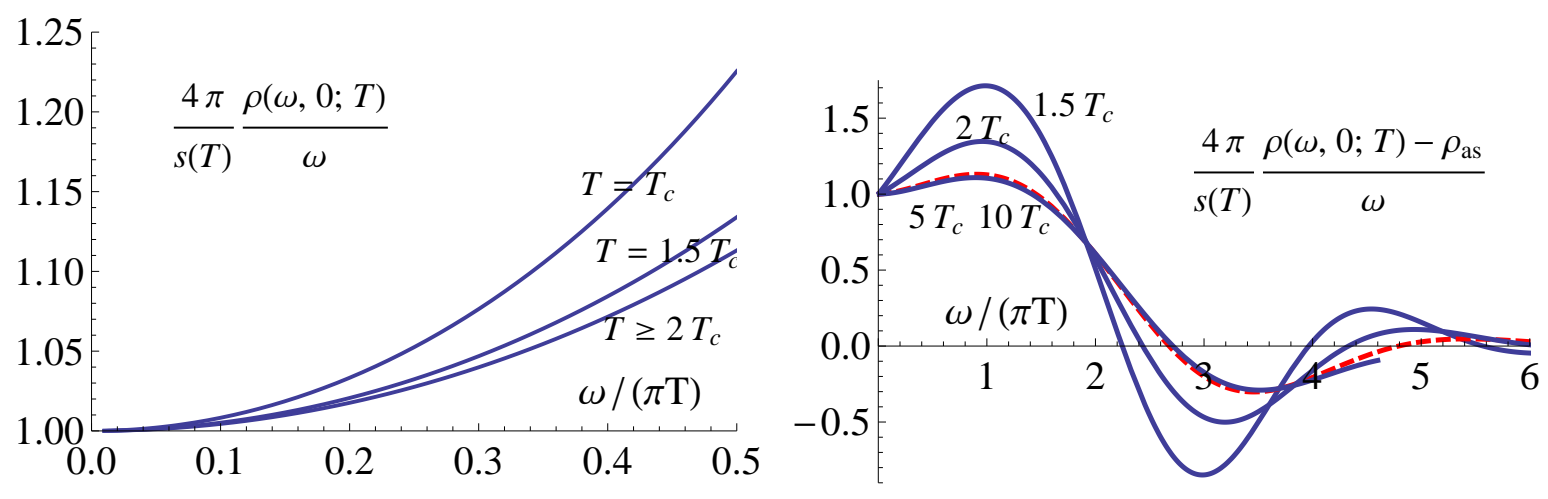

Figure 7: The imaginary part of the Green's function $G(\omega, 0 ; T)$ normalised as in 80 plotted vs $\omega /(\pi T)$ for small $\omega$ (left panel) or larger $\omega$ with the asymptotic behavior (76) subtracted (right panel). The subtraction is reliable only for $\omega /(\pi T) \gtrsim 5.0 T_{c} /(\pi T)$. The model $(15)$ is used here.

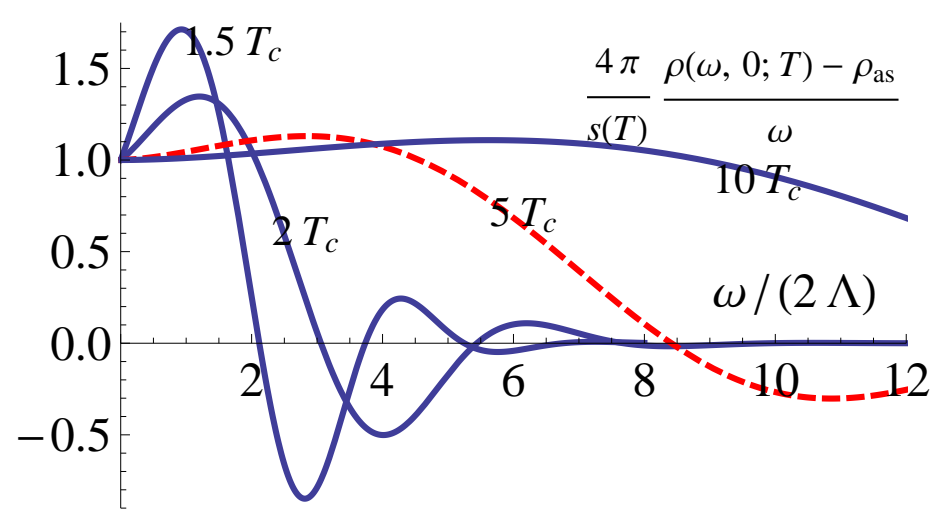

Figure 8: The imaginary part of the Green's function $G(\omega, 0 ; T)$ with the asymptotic behavior subtracted, normalised as in 80 plotted vs $\hat{\omega}=\omega /(2 \Lambda)$. The model 15 is used here.

peaks in (72). One may try to define a glueball mass at finite temperature by the position of the first peak in this curve. This grows very accurately linearly with $T$ :

$$
\hat{m}_{G}(T)=\frac{m_{G}(T)}{m_{\mathrm{G}} / \sqrt{2}}=1.016 \frac{T}{T_{c}}-0.241,
$$

where the lowest tensor glueball mass is $m_{G}=2 \sqrt{2} \Lambda$. 

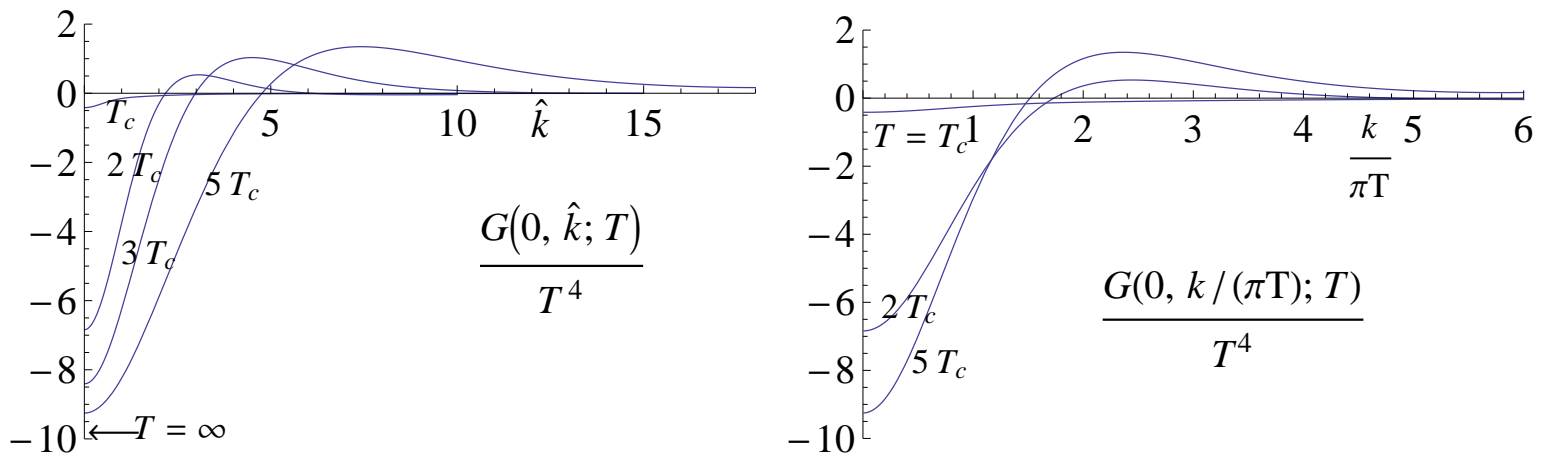

Figure 9: The (real) static Green's function $G(0, k ; T) / T^{4}$ in units of $\mathcal{L}^{3} /\left(4 \pi G_{5}\right)$ with the asymptotic behavior 75 subtracted plotted vs $\hat{k}=k /(2 \Lambda)$ (left panel) or vs $k /(\pi T)$ (right panel).

\subsection{Real static correlator, $\omega=0$}

The static correlator is defined by

$$
G(r)=\int_{0}^{\beta} d \tau\left\langle T_{12}(\tau, \mathbf{x}) T_{12}(0, \mathbf{0})\right\rangle_{T}=\int_{0}^{\beta} d \tau G(\tau, \mathbf{x})=\frac{1}{2 \pi^{2} r} \int_{0}^{\infty} d k k \sin (r k) G(k)
$$

and can also be related to the $\omega$-dependent spectral function by

$$
G(k)=\int_{0}^{\infty} \frac{d \omega}{\pi} \frac{2 \rho(\omega, k)}{\omega} .
$$

It is determined numerically by solving (39) with $\omega=0$ so that the boundary condition at the horizon is (41) with $\omega=0$ and solving $G(0, k)$ from (52) as a ratio of two Wronskians. It is also important to eliminate the large- $k$ behavior given in $(75)$, including the constant term. Upon transforming to coordinate space these give rise to $\delta(\mathbf{x})$ or its derivatives. To get $G(0, k ; T) / T^{4}$ one further has to use 65$)$.

Subtracting the expansion 75 from the numerically computed $B / A$ gives the result in Fig. 9 for the static correlator. The curves are of the same shape as those in the conformal case [8], where one plots vs $k /(\pi T)$. When $T \rightarrow \infty$ the value at $k=0$ of the curves approach the value $-\pi^{4} / 10$ of the conformal case. The dominant scale breaking effect is the rapid decrease of the correlator when $T$ decreases towards $T_{c}$. The next subsection explains how this is via Eq. (83) related to the behavior of $\rho(\omega, k)$ near the light cone, see Fig. 12 .

In the conformal case one can define a second order dissipative coefficient by the expansion

$$
G(0, k ; T)=G_{0}+\frac{1}{2} \kappa(T) k^{2}+\mathcal{O}\left(k^{4}\right)
$$

and its value is [40]

$$
\kappa=\frac{\mathcal{L}^{3}}{4 \pi G_{5}} \frac{1}{4} \pi^{2} T^{2}=\frac{1}{8} N_{c}^{2} T^{2}=\frac{s}{4 \pi^{2} T} .
$$




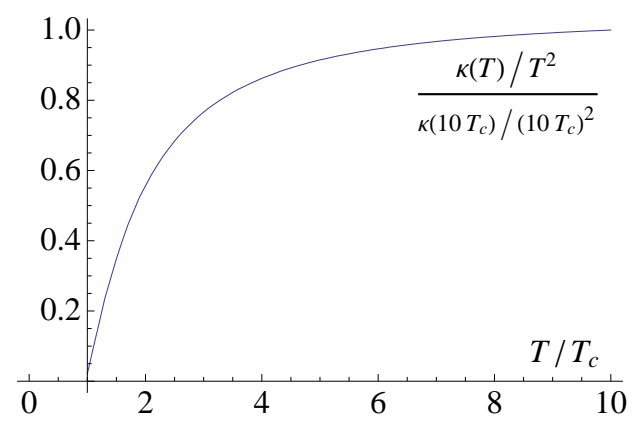

Figure 10: The expansion coefficient $\kappa(T)$ defined by $\sqrt{10}$, normalised to the value of $\kappa(T) / T^{2}$ at $10 T_{c}$.

Inserting the normalisation (30), our computation of $\kappa$ at $10 T_{c}$ is $1 \%$ from the conformal value in 85 . At smaller $T$ there is additional suppression on top of the $T^{2}$ dependence. This is quantitatively shown in Fig. 10 .

Given the momentum dependent static Green's function $G(k)$, the coordinate dependent correlator $G(r)$ can be computed by carrying out the integral 82 . The shape of the curves is very similar to that plotted explicitly for the conformal case in [8].

\subsection{Green's function for any $\omega, k$}

The dominant feature on the $\omega, k$ plane is the structure around the light cone. In [8] this was analysed analytically and, for example, one could prove that exactly on the light cone

$G(k, k)=(1+i \sqrt{3}) \frac{\Gamma(1 / 3)}{8 \cdot 6^{1 / 3} \Gamma(2 / 3)} k^{4 / 3}+\mathcal{O}(1) \approx(0.136092+i 0.235718) k^{4 / 3}, \quad k \equiv k /(\pi T)$.

Results for the spectral function as a function of $\omega$ at fixed values of $k$ are shown in Fig. 11 . The left panel shows what effects changing $T$ at fixed value of $k /(\pi T)$ has: the correlation function monotonically decreases. The right panel shows that essentially the conformal set of curves is obtained for $T=10 T_{c}$, for the model (15).

Another useful way of analysing the light cone region is to see how the spectral function varies when crossing the light cone perpendicular to it, i.e. at fixed $\omega_{+}=(\omega+k) / \sqrt{2}$. The outcome is shown in Fig. 12 at a fixed value of $\omega_{+}$. At larger $\omega_{+}$the peak gets narrower $\sim 1 / k^{1 / 3}$ but higher $\sim k^{1 / 3}$ so that the integral over $\omega$, which via Eq. 83 leads to the static correlator, is essentially constant. This is as shown in [8] for the conformal case. The new element is that when $T$ decreases towards $T_{c}$, the correlator also decreases. After integration over $\omega$ according to 83 this correlates with the rapid decrease of the static correlator, already shown in Fig. 9 . 

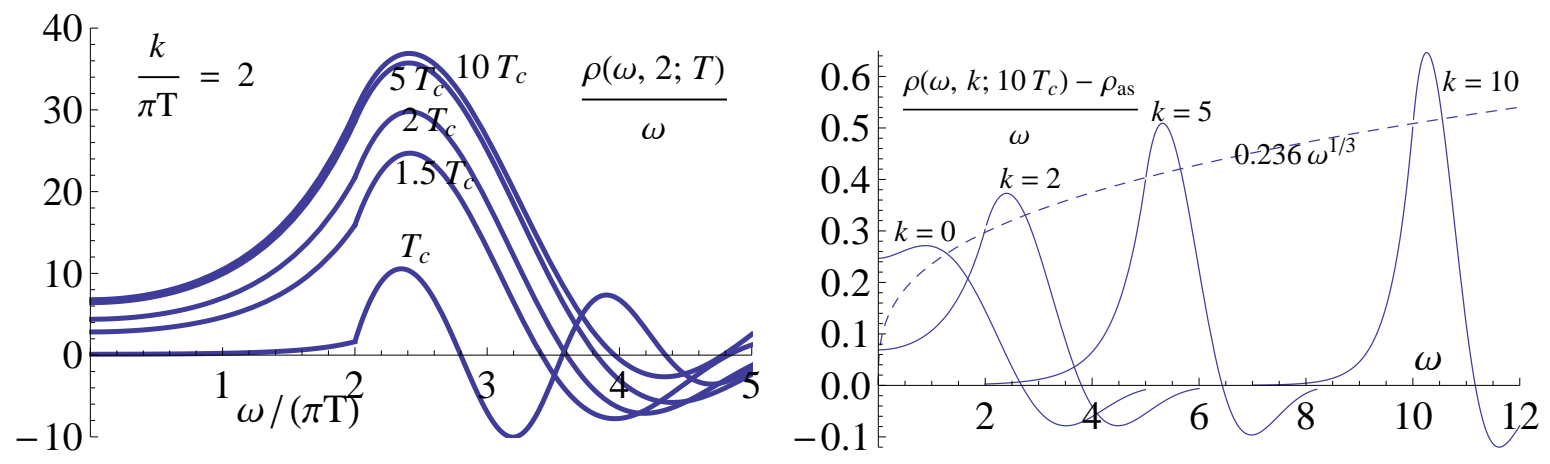

Figure 11: The imaginary part of the Green's function $G(\omega, k=2 \pi T ; T) / T^{4}$ in units of $\mathcal{L}^{3} /\left(4 \pi G_{5}\right)$ plotted vs $\omega /(\pi T)$, for the model (15). The right panel shows the behavior near the peaks at $k=$ $2,5,10 \pi T$. The curve $0.236 \omega^{1 / 3}$ is the conformal behavior of the correlator on the light cone, $\omega=k$.

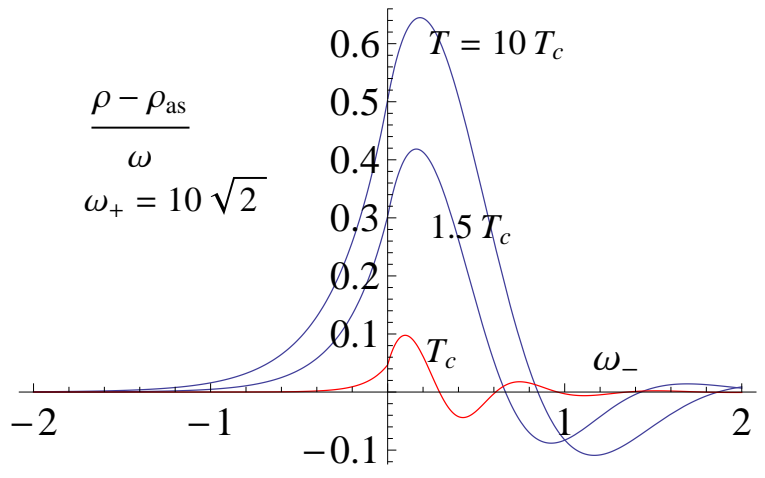

Figure 12: The quantity $\left(\rho(\omega, k ; T)-\rho_{\text {as }}(\omega, k)\right) / \omega$ when crossing the light cone in the perpendicular direction, i.e., at fixed $\omega_{+}=(\omega+k) / \sqrt{2}$ (here $\omega_{+}=10 \sqrt{2}$ ) with varying $\omega_{-}$, for various temperatures.

\section{Conclusions}

We have in this paper computed the retarded Green's function $G(\omega, k ; T), \mathbf{k}=(0,0, k)$, of the 1,2 component of the energy momentum tensor in the high temperature phase of a QCD-like strongly coupled theory with a first order phase transition and glueballs, using gauge/gravity duality. Temperatures between $T_{c}$ and $10 T_{c}$ were numerically studied. Results were given for the $\omega$ dependence of the spectral function at $k=0$, from which one could compute the $T$ dependence of the imaginary time correlator $G(\tau ; T)$, measured on the lattice. The real static correlator at $\omega=0$ was also computed and related to structure observed near the light cone, $\omega \approx k$. Overall, at $10 T_{c}$ the correlators were very close to the conformal situation [8], but large effects were observed when $T$ decreased towards $T_{c}$.

The gravity background used here is IHQCD as developed in [17]-[20] for $k=0$ and a 
simplified and quasianalytically tractable version thereof for all $\omega, k$. IHQCD describes very successfully the properties of large $N_{c}$ Yang-Mills theory both at $T=0$ and at finite $T$, but its gravity background, in general, has to be evaluated numerically. The model used here retains the attractive features of IHQCD in the infrared, so that it has confinement, glueballs and a first order phase transition, while simplifying the complicated logarithmic structure of IHQCD in the ultraviolet. The chief virtue of the model is that both $b(z)$ and $f(z)$, appearing in the fluctuation equation, are analytically known. Another analytic approximation to IHQCD [24, 41], would be quantitatively more accurate but does not permit analytic evaluation of $f(z)$. In any case, we expect that the results of this paper should be qualitatively valid for IHQCD, too, for all $\omega, k$. This is confirmed by numerical computations at $k=0$.

There is ample phenomenological evidence for the strongly interacting nature of QCD matter at $T \gtrsim T_{c}$ ( $p=\frac{3}{4} p_{\text {ideal }}$, small viscosity, jet quenching), but one has not been able to develop a first-principle numerically verifiable criterion for this. We have suggested that a quantitative verification of our predictions could serve this purpose.

There are several obvious directions of further study. For the first, a full numerical computation in IHQCD should be carried out, also for nonzero $k$. This is technically complicated due to the fact that the expansions near the boundary are not power series in $z$ but contain powers of $\log z$, studied here in Appendix A. Secondly, to extend the calculation to correlators of the scalar operator $\operatorname{Tr} F_{\mu \nu}^{2}$ one should use the more complicated fluctuation equation in [23], coupling the dilaton and the scalar component of the tensor fluctuation. A third open problem is the computation of more terms in the large $\omega$ expansion (74). Finally, it will be interesting to compare the results with those from next-to-leading order perturbative QCD, when they are available.

Acknowledgements. We thank J. Alanen, Jorge Casalderrey-Solana, Mikko Laine and Francesco Nitti for discussions and advice. F. Nitti has given us the Mathematica code used in [20]. The work of MV has been supported by Academy of Finland, contract no. 128792 and that of MK and AV by the Sofja Kovalevskaja program of the Alexander von Humboldt foundation. KK and AV thank the 2010 ESI workshop "AdS Holography and Quark-Gluon Plasma" in Vienna for hospitality.

\section{A Appendix: UV expansion in IHQCD}

In this appendix, we shall give small- $z$ logarithmic expansions of $b(z), \lambda(z)$ and $W(z) \equiv-\dot{b} / b^{2}$, adding a few terms to those given in Appendix A of [17]. Also $f(z)$ will obtain similar logarithmic corrections to the $z^{4}$ term by integration of 20 . 
The expansions are given as powers of $\lambda(z)$ or as inverse powers of

$$
L \equiv \log \frac{1}{\Lambda z}
$$

where $\Lambda$ is the integration constant of

$$
\frac{d \lambda}{d z}=-\beta W b(z)
$$

The correction terms arise from the UV beta function expansion written in the form

$$
b_{0} \beta(\lambda)=-\left(b_{0} \lambda\right)^{2}-\frac{b_{1}}{b_{0}^{2}}\left(b_{0} \lambda\right)^{3}-\frac{b_{2}}{b_{0}^{3}}\left(b_{0} \lambda\right)^{4}-\frac{b_{3}}{b_{0}^{4}}\left(b_{0} \lambda\right)^{5}+. .
$$

where only $b_{0}, b_{1}$ are scheme independent. It will appear that all quantities are functions of

$$
b_{0} \lambda, \quad \frac{b_{i}}{b_{0}^{i+1}}, \quad b \equiv \frac{b_{1}}{b_{0}^{2}}
$$

$\left(b \equiv b_{1} / b_{0}^{2}\right.$ is a standard notation, not to be confused with $\left.b(z)\right)$.

First, by integrating $d b / b=d \lambda / \beta(\lambda)=d b_{0} \lambda /\left(b_{0} \beta(\lambda)\right.$ ), (note the different constants $\hat{b}_{0}$ and $\left.b_{0}\right)$

$$
\frac{b}{\hat{b}_{0}}=\exp \left(\frac{1}{b_{0} \lambda}\right)\left(b_{0} \lambda\right)^{b_{1} / b_{0}^{2}}\left\{1+\left(\frac{b_{2}}{b_{0}^{3}}-b^{2}\right) b_{0} \lambda+\left[b(1+b)\left(b^{2}-2 \frac{b_{2}}{b_{0}^{3}}\right)+\frac{b_{2}^{2}}{b_{0}^{6}}+\frac{b_{3}}{b_{0}^{4}}\right] \frac{1}{2}\left(b_{0} \lambda\right)^{2}+. .\right\} .
$$

The constant of integration $\hat{b}_{0}$ is fixed so that $b_{0} \lambda$ appears in the powerlike term.

For $W \equiv-\dot{b} / b^{2}$ one can derive

$$
W(\lambda)=W(0) \exp \left(-\frac{4}{9} \int_{0}^{\lambda} d \bar{\lambda} \frac{\beta(\bar{\lambda})}{\bar{\lambda}^{2}}\right), \quad W(0)=\frac{1}{\mathcal{L}}
$$

Expanding this one has

$$
\mathcal{L} W=1+\frac{4}{9} b_{0} \lambda+\left(\frac{8}{81}+\frac{2}{9} \frac{b_{1}}{b_{0}^{2}}\right)\left(b_{0} \lambda\right)^{2}+4\left(\frac{8}{3^{7}}+\frac{2}{81} \frac{b_{1}}{b_{0}^{2}}+\frac{1}{27} \frac{b_{2}}{b_{0}^{3}}\right)\left(b_{0} \lambda\right)^{3}+. .
$$

The $z$ dependence is obtained by combining the equations $W=-\dot{b} / b^{2}$ and $\beta=b \dot{\lambda} / \dot{b}$ into (88) so that, integrating,

$$
z=\int_{0}^{z} d z=\int_{0}^{\lambda(z)} \frac{d \lambda}{-\beta W b}
$$

Noting that $W$ and $b$ contain the constants $1 / \mathcal{L}$ and $\hat{b}_{0}$ this equation naturally defines the scale

$$
\Lambda=\frac{\hat{b}_{0}}{\mathcal{L}}
$$


of $z$. Eq. (94) now has to be inverted for $\lambda$ : one expands the RHS around $\lambda=0$ using the expansions above, integrates term by term and takes the limit $\lambda \rightarrow 0$. Finally, the result is inverted recursively to obtain $\lambda=\lambda(z)$. The expansion one obtains is

$$
\begin{aligned}
& b_{0} \lambda=\frac{1}{L}-\frac{b \log L}{L^{2}}+\left(b^{2} \log ^{2} L-b^{2} \log L+\frac{4}{9}-b^{2}+\frac{b_{2}}{b_{0}^{3}}\right) \frac{1}{L^{3}}+ \\
+ & {\left[-b^{3} \log ^{3} L+\frac{5}{2} b^{3} \log ^{2} L-\left(3 b \frac{b_{2}}{b_{0}^{3}}-2 b^{3}+\frac{4}{3} b\right) \log L-\frac{4}{9}+\frac{2}{3} b-\frac{1}{2} b^{3}+\frac{b_{3}}{2 b_{0}^{4}}\right] \frac{1}{L^{4}}+} \\
+ & {\left[b^{4} \log ^{4} L-\frac{13}{3} b^{4} \log ^{3} L+\left(\frac{3}{2} b^{4}+\frac{8}{3} b^{2}+6 b^{2} \frac{b_{2}}{b_{0}^{3}}\right) \log ^{2} L+\left(4 b^{4}-4 b^{2}+\frac{16}{9} b+3 b^{2} \frac{b_{2}}{b_{0}^{3}}-2 b \frac{b_{3}}{b_{0}^{4}}\right) \log L\right.} \\
+ & \left.\frac{104}{81}-\frac{4}{3} b-\frac{10}{9} b^{2}+\frac{7}{6} b^{4}+\frac{52}{27} \frac{b_{2}}{b_{0}^{3}}-3 b^{2} \frac{b_{2}}{b_{0}^{3}}+\frac{5}{3} \frac{b_{2}^{2}}{b_{0}^{6}}-\frac{1}{6} b \frac{b_{3}}{b_{0}^{4}}+\frac{1}{3} \frac{b_{4}}{b_{0}^{5}}\right] \frac{1}{L^{5}}+\mathcal{O}\left(\frac{1}{L^{6}}\right) .
\end{aligned}
$$

To get corrections to $b(z)$ one writes $(88)$ in the form

$$
b(z)=\frac{\lambda^{\prime}(z)}{-\beta W}, \quad \lambda^{\prime}(z)=-\frac{1}{z} \frac{d \lambda}{d L},
$$

uses (96) and expands in $\lambda$. This leads to

$$
\begin{aligned}
b(z) & =\frac{\mathcal{L}}{z}\left[1-\frac{4}{9} b_{0} \lambda+\left(\frac{44}{81}-\frac{2}{9} b\right)\left(b_{0} \lambda\right)^{2}-\left(\frac{2408}{2187}-\frac{80}{81} b+\frac{4}{27} \frac{b_{2}}{b_{0}^{3}}\right)\left(b_{0} \lambda\right)^{3}+\mathcal{O}\left(\lambda^{4}\right)\right] \\
& =\frac{\mathcal{L}}{z}\left\{1-\frac{4}{9 L}+\frac{2(18 b \log L+22-9 b)}{81 L^{2}}+\right. \\
-4 & {\left.\left[\frac{710}{2187}-\frac{20}{81} b-\frac{1}{9} b^{2}+\frac{4}{27} \frac{b_{2}}{b_{0}^{3}}+\left(\frac{22}{81} b-\frac{2}{9} b^{2}\right) \log L+\frac{1}{9} b^{2} \log ^{2} L\right] \frac{1}{L^{3}}+\mathcal{O}\left(\frac{1}{L^{4}}\right)\right\} . }
\end{aligned}
$$

The three first terms in the second form were given in (A.28) of [17].

Finally, the UV expansion of the potential $\mathrm{V}$ is very simply directly obtained by inserting the expansions of $W$ and $\beta$ to $V(\lambda)=12 W^{2}(\lambda)\left[1-(\beta /(3 \lambda))^{2}\right]$ :

$$
V=\frac{12}{\mathcal{L}^{2}}\left[1+\frac{8}{9} b_{0} \lambda+\left(\frac{23}{81}+\frac{4}{9} b\right)\left(b_{0} \lambda\right)^{2}+\left(\frac{40}{2187}+\frac{14}{81} b+\frac{8}{27} \frac{b_{2}}{b_{0}^{3}}\right)\left(b_{0} \lambda\right)^{3}+\mathcal{O}\left(\lambda^{4}\right)\right] .
$$

\section{B Appendix: IR expansion}

One cannot uniquely solve the equations (8) at large $z$, large $\lambda$, but assume that $b(z)$ behaves as

$$
b(z)=b_{0} e^{-(\Xi z)^{\alpha}}(\Xi z)^{p}, \quad \alpha \geq 1, \quad p \text { real. }
$$

$\Xi$ here in the IR is a number times $\Lambda$ in the UV and, to emphasize this difference, we use a different letter for it. Inserting this to the equations of motion above and evaluating successively $\dot{b} / b, b W, W, \dot{W} / W$ one can write $\dot{\phi}$ in a form which is easily integrable. If $\lambda_{0}$ is 
the constant of integration of this equation, one finds that, up to corrections $1+\mathcal{O}\left(1 / z^{\alpha}\right) \sim$ $1+\mathcal{O}(1 / \log \lambda)$

$$
\begin{aligned}
b(z)= & b_{0} e^{-(\Xi z)^{\alpha}}(\Xi z)^{p}=\frac{b_{0}}{\lambda^{2 / 3}}\left(\frac{2}{3} \log \lambda\right)^{(\alpha-1) /(2 \alpha)}, \\
W(z)= & \frac{\alpha}{\mathcal{L}} e^{(\Xi z)^{\alpha}}(\Xi z)^{\alpha-1-p}=\frac{1}{\mathcal{L}} \lambda^{2 / 3} \alpha\left(\frac{2}{3} \log \lambda\right)^{(\alpha-1) /(2 \alpha)}, \quad \mathcal{L}=\frac{b_{0}}{\Lambda}, \\
\lambda(z)= & e^{3(\Xi z)^{\alpha} / 2}(\Xi z)^{3(\alpha-1-2 p) / 4}, \\
& e^{(\Xi z)^{\alpha}}=\lambda^{2 / 3}(\Lambda z)^{p-\alpha / 2+1 / 2}, \Xi z=\left(\frac{2}{3} \log \lambda\right)^{1 / \alpha}, \\
\beta(\lambda)= & -\frac{3}{2} \lambda\left(1+\frac{\alpha-1}{2 \alpha(\Xi z)^{\alpha}}+\mathcal{O}\left(\frac{1}{z^{2 \alpha}}\right)\right)=-\frac{3}{2} \lambda\left(1+\frac{3(\alpha-1)}{4 \alpha \log \lambda}+\mathcal{O}\left(\frac{1}{\log ^{2} \lambda}\right)\right), \\
V(z)= & 9 W^{2}(z)=\frac{9 \alpha^{2}}{\mathcal{L}^{2}} e^{2(\Xi z)^{\alpha}}(\Xi z)^{2(\alpha-1-p)}=\frac{9 \alpha^{2}}{\mathcal{L}^{2}} \lambda^{4 / 3}\left(\frac{2}{3} \log \lambda\right)^{(\alpha-1) /(\alpha)},
\end{aligned}
$$

where always $\lambda \equiv \lambda / \lambda_{0}$, apart from the leading term in $\beta(\lambda)$, where the leading term is $-3 \lambda / 2$ for any $\lambda_{0}$. By choosing $p$ suitably one can make any of the quantities a pure exponential in $z^{\alpha}$. However, when expressed in terms of $\lambda$, the leading behavior is always independent

of $p$. Note that $b \lambda^{2 / 3} \equiv b_{s} \sim(\log \lambda)^{(\alpha-1) /(2 \alpha)}$ is the string frame $b$, which is used to express the confinement criterion. Note also that the confining beta function is not assumed here, it follows from the assumed form of $b(z)$. The scalar potential $V$ satisfies $V=12 W^{2}\left(1-X^{2}\right)$, $X \equiv \beta /(3 \lambda)$.

Finally, Eq. (20) now is

$$
\dot{f}(z)=C e^{3(\Xi z)^{\alpha}}(\Xi z)^{-3 p} .
$$

In our model with $\alpha=2, p=-1$ this is applies at all $z$ and is simply integrable in closed form. If one wants just a large $z$ approximation for $f(z)$ one can integrate it up to corrections of order $1 / z^{\alpha}$ by extending the integration to $z=0$ and imposing the condition $f(0)=1$. This leads to

$$
\begin{aligned}
f(z) & =1-\left(\frac{z}{z_{h}}\right)^{1-\alpha-3 p} e^{3 \Xi^{\alpha}\left(z^{\alpha}-z_{h}^{\alpha}\right)}, \\
-\dot{f}\left(z_{h}\right) & =4 \pi T=3 \alpha \Xi\left(\Xi z_{h}\right)^{\alpha-1} .
\end{aligned}
$$

\section{Ultraviolet limit of the static correlator}

In this appendix we derive the high- $k$ limit the static correlator $G(\omega=0, \mathbf{k} ; T)$. Eliminating the first derivative in (53) by writing

$$
\psi(y)=\sqrt{b^{3} f \sqrt{y}} \cdot \phi(y)
$$

and setting $\omega=0$, we have the equation

$$
\psi^{\prime \prime}(y)=\left[\frac{\hat{k}^{2}}{y f(y)}-\left(\frac{f^{\prime}(y)}{2 f(y)}\right)^{2}+\frac{1}{4}+\frac{1}{2 y}+\frac{3}{4 y^{2}}\right] \psi(y) \equiv\left[\hat{k}^{2} F(y)+G(y)\right] \psi(y)=0
$$


We wish to solve this around $y=0$ for $\hat{k} \rightarrow \infty$. The systematic approach given in [30] starts by defining

$$
\xi \equiv \int_{0}^{y} \frac{d x}{\sqrt{x f(x)}}, \quad \psi(y)=\left(\frac{d \xi^{2}}{d y}\right)^{-1 / 2} W(\xi) .
$$

The idea with replacing $y$ by the new variable $\xi$ is that the resulting equation is in a Schrödinger-like form in which only $k^{2}$ multiplies the function to be solved. Introducing $W$ eliminates the first derivative generated. The solution then is given in the form 3

$$
W(\xi)=\xi K_{2}(\hat{k} \xi) \sum_{s=0}^{\infty} \frac{A_{s}(\xi)}{\hat{k}^{2 s}}-\frac{\xi^{2}}{\hat{k}} K_{3}(\hat{k} \xi) \sum_{s=0}^{\infty} \frac{B_{s}(\xi)}{\hat{k}^{2 s}},
$$

where the coefficients $A_{s}$ and $B_{s}$ are computed from the corresponding expansion coefficients $\mathrm{A}_{\mathrm{s}}$ describing the solution away from the singular point $\xi=0$ :

$$
A_{s}(\xi)=\sum_{j=0}^{2 s} a_{j}(3) \xi^{-j} \mathrm{~A}_{2 s-j}(\xi), \quad B_{s}(\xi)=\sum_{j=0}^{2 s+1} a_{j}(2) \xi^{-j-1} \mathrm{~A}_{2 s-j-1}(\xi),
$$

where $a_{j}(\nu)=\prod_{k=1}^{j}\left(4 \nu^{2}-(2 k-1)^{2}\right) / 8 k$. Functions $\mathrm{A}_{s}$ in turn are given by

$$
\begin{aligned}
\mathrm{A}_{s+1}(\xi) & =-\frac{1}{2} \mathrm{~A}_{s}^{\prime}(\xi)+\frac{1}{2} \int d \xi \Psi(\xi) \mathrm{A}_{s}(\xi) \\
& =-\frac{1}{2} \sqrt{y f(y)} \mathrm{A}_{s}^{\prime}(y)+\frac{1}{2} \int \Psi(y) \mathrm{A}_{s}(y) \frac{d y}{\sqrt{y f(y)}}
\end{aligned}
$$

The integration constants in each step are chosen so that when we retrace our steps back to the original mode function $\phi(y)$, we have $\phi(0)$ finite and equal to 1 . The function $\Psi(y)$ in the above recursion relation is given by the coefficient functions $F(y), G(y)$ of eq. (109), and reads

$$
\Psi(y)=\frac{G(y)}{F(y)}-F(y)^{-3 / 4} \frac{d^{2}}{d y^{2}} F(y)^{-1 / 4} \approx \frac{15}{16 y}+\frac{1}{2}+\frac{8 y_{h}-8+5 e^{-y_{h}}}{32\left(y_{h}-1+e^{-y_{h}}\right)} y+\ldots
$$

Working out the series expansions of $\mathrm{A}_{s}, s=0 \ldots 4$ (with $\mathrm{A}_{s}=$ constant), we can read out the coefficients of the $y^{0}$ and $y^{2}$ terms in

$$
\phi(y)=\left(b^{3} f \sqrt{y}\right)^{-1 / 2} \psi(y)=\left(2 \xi b^{3} \sqrt{f}\right)^{-1 / 2} w(\xi)
$$

to finally arrive at

$$
\frac{B}{A}=-\hat{k}^{4}\left(\ln \hat{k}+\gamma_{E}-\frac{3}{4}\right)-\hat{k}^{2}\left(\ln \hat{k}+\gamma_{E}\right)-\frac{5}{24}+\frac{e^{-y_{h}}}{20\left(y_{h}-1+e^{-y_{h}}\right)} .
$$

\footnotetext{
${ }^{3}$ For the coefficients $A_{s}$ and $B_{s}$, we follow here Exercise 5.2 of Chapter 12 in 30 .
} 


\section{References}

[1] G. Policastro and A. Starinets, "On the absorption by near-extremal black branes," Nucl. Phys. B 610, 117 (2001) arXiv:hep-th/0104065.

[2] D. T. Son and A. O. Starinets, "Minkowski-space correlators in AdS/CFT correspondence: Recipe and applications," JHEP 0209, 042 (2002) arXiv:hep-th/0205051.

[3] P. K. Kovtun and A. O. Starinets, "Quasinormal modes and holography," Phys. Rev. D 72, 086009 (2005) arXiv:hep-th/0506184.

[4] P. Kovtun and A. Starinets, "Thermal spectral functions of strongly coupled N = 4 supersymmetric Yang-Mills theory," Phys. Rev. Lett. 96, 131601 (2006) arXiv:hepth/0602059.

[5] D. Teaney, "Finite temperature spectral densities of momentum and R-charge correlators in N=4 Yang Mills theory," Phys. Rev. D74, 045025 (2006). hep-ph/0602044.

[6] S. S. Gubser, S. S. Pufu, F. D. Rocha, JHEP 0808, 085 (2008). arXiv:0806.0407 [hepth]].

[7] P. Romatschke and D. T. Son, "Spectral sum rules for the quark-gluon plasma," Phys. Rev. D 80, 065021 (2009) arXiv:0903.3946 [hep-ph]].

[8] K. Kajantie, M. Vepsalainen, "Spatial scalar correlator in strongly coupled hot N=4 Yang-Mills theory," Phys. Rev. D83, 066003 (2011). arXiv:1011.5570 [hep-th]].

[9] M. Laine, M. Vepsalainen and A. Vuorinen, "Ultraviolet asymptotics of scalar and pseudoscalar correlators in hot Yang-Mills theory," JHEP 1010, 010 (2010) arXiv:1008.3263 [hep-ph]].

[10] M. Laine, M. Vepsalainen and A. Vuorinen, "Intermediate distance correlators in hot Yang-Mills theory," arXiv:1011.4439 [hep-ph].

[11] H. B. Meyer, "Energy-momentum tensor correlators and spectral functions," JHEP 0808, 031 (2008) [arXiv:0806.3914 [hep-lat]].

[12] N. Iqbal and H. B. Meyer, "Spatial correlators in strongly coupled plasmas," JHEP 0911, 029 (2009) arXiv:0909.0582 [hep-lat]].

[13] T. Springer, C. Gale, S. Jeon, S. H. Lee, "A shear spectral sum rule in a non-conformal gravity dual," Phys. Rev. D82, 106005 (2010). arXiv:1006.4667 [hep-th]].

[14] E. Iancu, A. H. Mueller, Phys. Lett. B681, 247-252 (2009). arXiv:0906.3175 [hep-ph]].

[15] M. Cheng, P. Hendge, C. Jung, F. Karsch, O. Kaczmarek, E. Laermann, R. D. Mawhinney, C. Miao et al., "Baryon Number, Strangeness and Electric Charge Fluctuations in QCD at High Temperature," Phys. Rev. D79, 074505 (2009). arXiv:0811.1006 [hep-lat]]. 
[16] T. Schafer, D. Teaney, "Nearly Perfect Fluidity: From Cold Atomic Gases to Hot Quark Gluon Plasmas," Rept. Prog. Phys. 72, 126001 (2009). arXiv:0904.3107 [hep-ph]].

[17] U. Gursoy and E. Kiritsis, "Exploring improved holographic theories for QCD: Part I," JHEP 0802, 032 (2008) arXiv:0707.1324 [hep-th]].

[18] U. Gursoy, E. Kiritsis and F. Nitti, "Exploring improved holographic theories for QCD: Part II," JHEP 0802, 019 (2008) arXiv:0707.1349 [hep-th]].

[19] U. Gursoy, E. Kiritsis, L. Mazzanti and F. Nitti, "Holography and Thermodynamics of 5D Dilaton-gravity," JHEP 0905, 033 (2009) [arXiv:0812.0792 [hep-th]].

[20] U. Gursoy, E. Kiritsis, L. Mazzanti and F. Nitti, "Improved Holographic Yang-Mills at Finite Temperature: Comparison with Data," Nucl. Phys. B 820, 148 (2009) arXiv:0903.2859 [hep-th]].

[21] E. Kiritsis and F. Nitti, "On massless 4D Gravitons from 5D Asymptotically AdS Spacetimes," Nucl. Phys. B 772, 67 (2007) arXiv:hep-th/0611344.

[22] T. Springer, "Sound Mode Hydrodynamics from Bulk Scalar Fields," Phys. Rev. D79, 046003 (2009). arXiv:0810.4354 [hep-th]].

[23] T. Springer, C. Gale, S. Jeon, "Bulk spectral functions in single and multi-scalar gravity duals," Phys. Rev. D82, 126011 (2010). arXiv:1010.2760 [hep-th]].

[24] J. Alanen, K. Kajantie, V. Suur-Uski, "A gauge/gravity duality model for gauge theory thermodynamics," Phys. Rev. D80, 126008 (2009). arXiv:0911.2114 [hep-ph]].

[25] A. Karch, E. Katz, D. T. Son and M. A. Stephanov, "Linear confinement and AdS/QCD," Phys. Rev. D 74, 015005 (2006) arXiv:hep-ph/0602229.

[26] E. Kiritsis, "Dissecting the string theory dual of QCD," Fortsch. Phys. 57, 396-417 (2009). arXiv:0901.1772 [hep-th]].

[27] A. Buchel, J. T. Liu, A. O. Starinets, "Coupling constant dependence of the shear viscosity in N=4 supersymmetric Yang-Mills theory," Nucl. Phys. B707, 56-68 (2005). hep-th/0406264.

[28] I. Papadimitriou, "Holographic renormalization as a canonical transformation," arXiv:1007.4592 [hep-th].

[29] H. Forkel, "Holographic glueball structure," Phys. Rev. D78, 025001 (2008). arXiv:0711.1179 [hep-ph]].

[30] F.W.J. Olver, Asymptotics and special functions, A.K.Peters, Wellesley, 1997.

[31] J. Noronha, "Transport Properties of QCD at Large N(c) and the Gauge / String Duality," arXiv:0912.4824 [hep-th]]. 
[32] See, for example, A. Mocsy, P. Petreczky, "Can quarkonia survive deconfinement?," Phys. Rev. D77, 014501 (2008). arXiv:0705.2559 [hep-ph]].

[33] F. Karsch, H. W. Wyld, "Thermal Green's Functions And Transport Coefficients On The Lattice," Phys. Rev. D35, 2518 (1987).

[34] G. Aarts, J. M. Martinez Resco, "Transport coefficients, spectral functions and the lattice," JHEP 0204, 053 (2002). [hep-ph/0203177].

[35] H. B. Meyer, "A Calculation of the shear viscosity in SU(3) gluodynamics," Phys. Rev. D76, 101701 (2007). arXiv:0704.1801 [hep-lat]].

[36] H. B. Meyer, "A Calculation of the bulk viscosity in SU(3) gluodynamics," Phys. Rev. Lett. 100, 162001 (2008). [arXiv:0710.3717 [hep-lat]].

[37] H. B. Meyer, "Energy-momentum tensor correlators and viscosity," PoS LATTICE2008, 017 (2008). arXiv:0809.5202 [hep-lat]].

[38] G. D. Moore, O. Saremi, "Bulk viscosity and spectral functions in QCD," JHEP 0809, 015 (2008). arXiv:0805.4201 [hep-ph]].

[39] H. B. Meyer, "Transport Properties of the Quark-Gluon Plasma - A Lattice QCD Perspective," arXiv:1104.3708 [hep-lat]].

[40] R. Baier, P. Romatschke, D. T. Son, A. O. Starinets, M. A. Stephanov, "Relativistic viscous hydrodynamics, conformal invariance, and holography," JHEP 0804, 100 (2008). arXiv:0712.2451 [hep-th]].

[41] A. Stoffers, I. Zahed, "Improved AdS/QCD Model with Matter," arXiv:1009.4428 [hepth]]. 